\title{
Mutations That Confer Drug-Resistance, Oncogenicity and Intrinsic Activity on the ERK MAP Kinases-Current State of the Art
}

\author{
Karina Smorodinsky-Atias ${ }^{1,+}$, Nadine Soudah ${ }^{1,+}$ and David Engelberg ${ }^{1,2,3, *}$ \\ 1 Department of Biological Chemistry, The Institute of Life Science, The Hebrew University of Jerusalem, \\ Jerusalem 91904, Israel; karinasun@gmail.com (K.S.-A.); nadine.soudah@mail.huji.ac.il (N.S.) \\ 2 CREATE-NUS-HUJ, Molecular Mechanisms Underlying Inflammatory Diseases (MMID), National \\ University of Singapore, 1 CREATE WAY, Innovation Wing, Singapore 138602, Singapore \\ 3 Department of Microbiology, Yong Loo Lin School of Medicine, National University of Singapore, Singapore \\ 117456, Singapore \\ * Correspondence: engelber@mail.huji.ac.il or MICDE@nus.edu.sg; Tel.: +972-2-6584718 or +972-54-2066378 \\ + These authors contributed equally to this work.
}

Received: 10 December 2019; Accepted: 2 January 2020; Published: 6 January 2020

\begin{abstract}
Unique characteristics distinguish extracellular signal-regulated kinases (Erks) from other eukaryotic protein kinases (ePKs). Unlike most ePKs, Erks do not autoactivate and they manifest no basal activity; they become catalysts only when dually phosphorylated on neighboring Thr and Tyr residues and they possess unique structural motifs. Erks function as the sole targets of the receptor tyrosine kinases (RTKs)-Ras-Raf-MEK signaling cascade, which controls numerous physiological processes and is mutated in most cancers. Erks are therefore the executers of the pathway's biology and pathology. As oncogenic mutations have not been identified in Erks themselves, combined with the tight regulation of their activity, Erks have been considered immune against mutations that would render them intrinsically active. Nevertheless, several such mutations have been generated on the basis of structure-function analysis, understanding of ePK evolution and, mostly, via genetic screens in lower eukaryotes. One of the mutations conferred oncogenic properties on Erk1. The number of interesting mutations in Erks has dramatically increased following the development of Erk-specific pharmacological inhibitors and identification of mutations that cause resistance to these compounds. Several mutations have been recently identified in cancer patients. Here we summarize the mutations identified in Erks so far, describe their properties and discuss their possible mechanism of action.
\end{abstract}

Keywords: MAPK kinase; ERK1; ERK2; CD domain; Rolled; SCH772984; VRT-11E; sevenmaker

\section{Introduction}

The unusual biochemical properties of the extracellular signal-regulated Kinases (Erks), their numerous biological functions and their critical roles in essentially all types of cancer, make these enzymes important subjects for research, and attractive targets for therapeutic purposes. Indeed, more than 50,000 studies have addressed aspects of the biochemistry, biology and pathology of Erks. Nevertheless, serious obstacles, which seem to be related to the unusual characteristics of the Erk enzymes, have been hindering the research. One of the hurdles has been the lack of key reagents, such as intrinsically/constitutively active mutants of Erks, and another is the absence of specific pharmacological inhibitors, not to mention clinically relevant inhibitors. The unavailability of these tools was unexpected, because useful inhibitors and a variety of active mutants were readily developed for most other protein kinases, including those that function upstream and downstream of Erk and those that are similar to Erks, such as p38s and JNKs [1-16]. 
This situation has been changing dramatically in the last decade. An arsenal of over a dozen useful inhibitors was finally developed, and, soon after, numerous mutations that render Erks resistant to these drugs were identified. Other mutations in ERKs were found in screens for cells in culture that acquired resistance to inhibitors of Raf and MEK. The mutations that cause drug resistance joined a small number of mutations that had been generated on the basis of gain-of-function mutations in lower organisms, or via structural studies. Finally, sequencing of genomes of tens of thousands of cancer patients led to the discovery of a few more mutations in ERKs.

Thus, a large number of interesting mutations in ERKs has been finally gathered (Table 1). The effects of many of these mutations on the structure, biochemistry, biology, or pathology of Erks have not yet been fully characterized, but some notions are emerging. This review summarizes our current knowledge of ERK mutations and describes their effect on the catalytic, physiological, pharmacological and pathological properties of Erks.

\subsection{The Erk MAP Kinases}

\subsubsection{The Erk MAP Kinases Are Conserved in All Eukaryotes and Carry Out a Plethora of Functions}

Erk proteins form a small subgroup within the family of MAP kinases. In mammals this group is encoded by two genes, ERK1 and ERK2, and by several splicing variants thereof. Erk1 and Erk2 are expressed in all cells of the organism and are critical for the functionality of all tissues and body systems. An indication of the remarkable competency of Erks is the large number of substrates they phosphorylate -497 have been identified so far [17]. For comprehensive reviews on the Erks, see [18].

Erks are highly conserved in evolution structurally and functionally, so that many discoveries with Erks' orthologs of S. cerevisiae (Fus3, Kss1 and Slt2/Mpk1; [19-22]) or of D. melanogaster (Rolled; [23,24]) are directly relevant to the mammalian molecules.

Mammalian Erk1 and Erk2 share 83\% sequence identity and 88\% similarity (alignment of the human proteins) and seem to be equally activated in response to relevant signals, suggesting that many of their activities are redundant. Observations that raised the possibility of distinct functions for each isoform were made primarily with knockout mice [25-34]. The most significant finding in this regard was that knocking out ERK2 resulted in embryonic lethality, whereas knocking out ERK1 had only mild effects [35]. Yet, overexpression of Erk1 in mice knocked-out for ERK2, restores viability and the mice are normal and fertile [36]. It seems, therefore, that the physiological functions of Erk1 and Erk2 are almost fully redundant and the dramatic difference in the phenotype between ERK1 $1^{-/}$and ERK2 ${ }^{-/-}$mice stems solely from the fact that in most tissues Erk2 is expressed at much higher levels than Erk1 [36]. Also pointing to similarity in structure-function relationships are the observations that the majority (but not all) of the mutations identified recently and discussed in this review confer similar effects on the Erk1 and Erk2 proteins. Finally, the newly developed pharmacological inhibitors manifest similar (but not identical) efficacy towards the two isoforms, although it should be noted that some of those inhibitors have not yet been tested against both isoforms. These observations combined suggest minor differences in functionality between Erk1 and Erk2 native proteins.

\subsubsection{Erks Are Targets of the Proto-Oncogenic RTK-Ras-Raf-MEK Pathway}

Erks function as the downstream targets of the receptor tyrosine kinase (RTK)-Ras-Raf-MEK pathway, which regulates a large number of biological processes in all cell types and in all developmental stages (for reviews on the RTK-Ras-Raf-MEK pathways see [37-41]). Although in particular cell-types and under some conditions Raf and MEK may phosphorylate various substrates [17], in response to most signals Erks seem to be the only targets of this cascade and therefore mediate most, if not all, of the effects of the pathway [42]. Erks are activated by all 20 subfamilies of RTKs [43], including the clinically important epidermal growth factor receptors (EGFRs), nerve growth factor receptors (NGFRs), vascular endothelial growth factor receptors (VEGFRs), platelet-derived growth factor receptors (PDGFRs), fibroblast growth factor receptors (FGFRs) and insulin receptors (InsRs) [43]. 
A series of consecutive reactions leads from ligand-bound receptor to Erk activation. Briefly, upon association with its ligand, the RTK dimerizes and trans-autophosphorylates on several tyrosine residues at its cellular domain [44]. The phosphorylated tyrosines serve as scaffolds for SH2- and PTB-containing cytoplasmic enzymes [38]. One of the protein complexes that bind to a phosphotyrosine on the RTK is Grb2-Sos, which in turn activates the small GTPase Ras. Active, GTP-bound, Ras recruits Raf proteins (A-Raf; B-Raf and c-Raf/Raf1) [45], the MAP3Ks of the Erk pathway. Raf kinases phosphorylate the MAP2Ks Mek1 and Mek2. Phosphorylated Meks dually phosphorylate Erk1/2 on neighboring Thr and Tyr residues, part of a TEY motif located at the activation loop. Several additional MAP3Ks may activate Mek, depending on the context of the cell and the type of stimulus (i.e., MOS [46], TPL2/Cot [47] and MLTK [48]). With only a few exceptions, Meks are the only known activators of Erk1/2. Without MEK-mediated dual phosphorylation, Erks are catalytically inactive. Erks can also be activated by GPCRs involving different subunits of G-proteins or $\beta$-arrestin, in a ligand-independent mechanism [49-52]. Thus, a variety of ligands, which activate either RTKs or GPCRs, as well as various environmental changes, lead to Erk activation. In addition to the interaction with the direct upstream activators, Erks interact with scaffold proteins such as KSR [53] and Mek partner-1 (MP-1) [54], which facilitate the association of the various cascade components thereby increasing the efficiency of their activation [55]. Activated Erks phosphorylate their substrates on Ser or Thr residues, in all cellular compartments. Cytoplasmic substrates include protein kinases, such as Rsk1/2, Mnk1/2 and Msk1 [56-58]. Nuclear targets include transcription factors of the Fos, Myc and Ets families [59]. Erks also phosphorylate upstream pathway components, such as Raf-1, B-Raf and Mek, as part of positive and negative feedback mechanisms [60-64]. Another manner of negative feedback is the Erk-induced expression of its own deactivating phosphatases [65]. For review on Erk substrates and downstream targets see [17].

\subsubsection{Erk Activation Is Achieved by Dual Phosphorylation of a TEY Motif within the Activation Loop}

The difficulty in obtaining mutations that render Erks intrinsically active may stem in part from its tight regulation, supported by unique structure-function properties that distinguish them from most other eukaryotic protein kinases (ePKs). Almost all ePKs share a common kinase domain, which includes a highly conserved ATP binding site, a catalytic site and an activation loop. ePKs reside in equilibrium between active and inactive conformations, so that these catalytically-relevant sites are functional only in the active conformation. The kinase domain of all ePKs, including Erks, consists of a small N-lobe and a larger C-lobe (Figure 1A). The N-lobe contains $5 \beta$-strands and a single helix ( $\alpha$ C-helix), which is dynamic and occupies the space between the lobes. The $\alpha C$-helix contains a conserved Glu, which, in the active conformation, forms a salt bridge with a Lys residue located within the AXK motif in $\beta 3$ strand. This bridge is important and conserved in all ePKs and ensures anchoring and proper orientation of the ATP molecule. The C-lobe, which is mainly $\alpha$-helical, binds and brings substrates adjacent to the ATP. A short (20-30 amino acids long) fragment located between the Nand C-lobes, known as the activation segment, contains some important elements, such as the DFG and APE motifs, the P+1 site, the catalytic and the activation loops (Figure 1A; [66]). The DFG motif is important for proper positioning of the ATP for phosphate transfer. While the Asp in the DFG is critical for recognizing the $\mathrm{Mg}^{+2}$ ions, the Phe forms hydrophobic interactions with the $\alpha \mathrm{C}$-helix and also with the catalytic Asp of the Y/HRD motif. The Y/HRD motif belongs to the catalytic loop responsible for catalysis. The conserved catalytic Asp of the Y/HRD motif functions by orienting the phosphate accepting hydroxyl as well as a proton-transfer acceptor. The Tyr/His residue of this motif, which is also conserved, is part of the R-spine and forms hydrophobic interactions with the DFG. An important conserved moiety is a phosphoacceptor (commonly a threonine) within the activation loop. In most ePKs, phosphorylation of this Thr is a pre-requisite for activity, as it is essential for shifting the equilibrium towards the active conformation. This phosphorylation induces several structural changes, including a conformational change of the DFG motif (to DFG 'in'), rotation of the 
$\alpha \mathrm{C}$-helix, which enables the formation of a Glu-Lys salt bridge, and a domain closure between the Nand C-lobes, which ultimately stabilize the regulatory and the catalytic spines of the enzyme $[67,68]$.

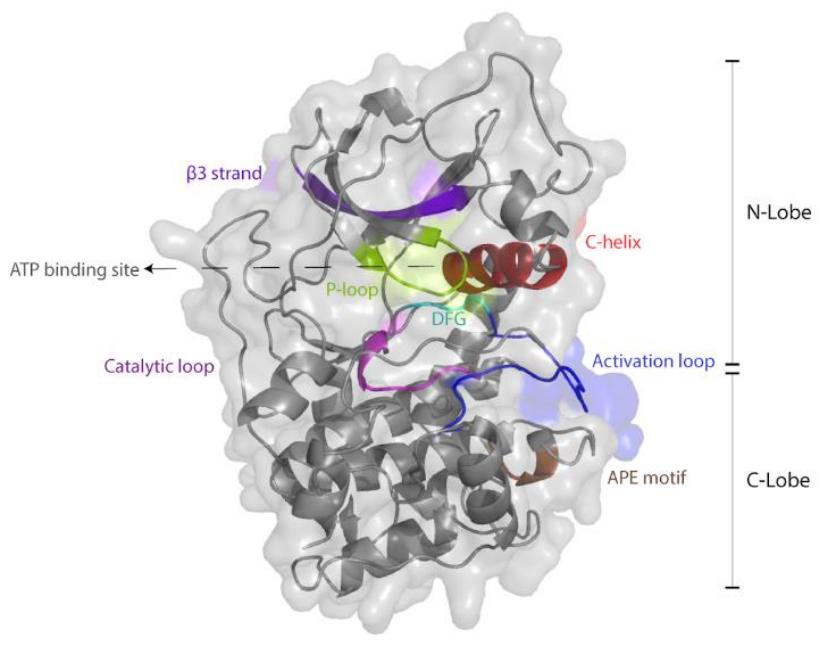

B

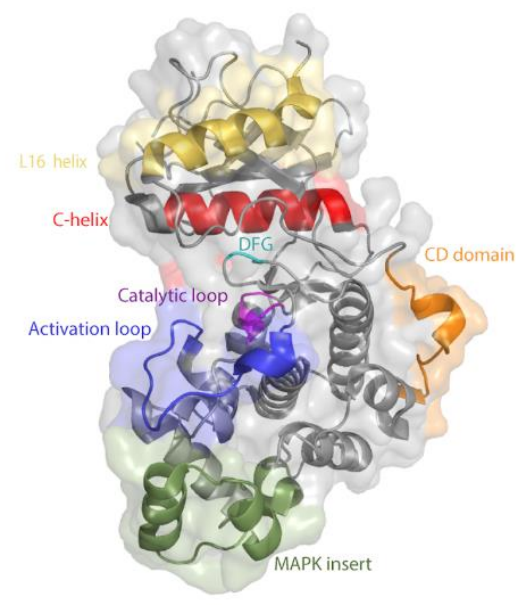

C

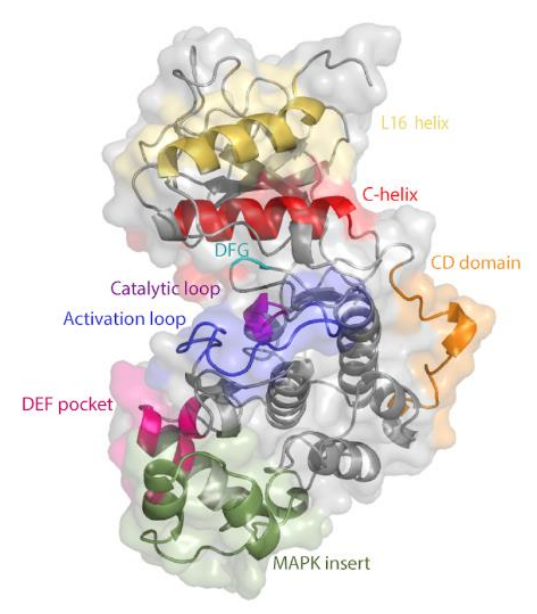

Figure 1. The kinase fold of Erks is highly similar to that of other ePKs, but they possess additional, specific domains. Shown are the crystal structures of (A) PKA (PDB 1FMO), (B) unphosphorylated Erk2 (PDB 4S31) and (C) dually phosphorylated Erk2 (PDB 2ERK). All panels show a cartoon representation covered with a transparent molecular surface with important regions presented and colored accordingly. Note the L16 helix and MAPK insert, not present in PKA, and the DEF pocket that forms only in phosphorylated Erk2.

As this dramatic shift from the non-active to active conformation is a result of the single phosphorylation event, the activation-loop phosphoacceptor Thr is an obvious target for regulation and for mutagenesis aimed at generating activating variants. In several ePKs, converting this Thr to Glu resulted in a constitutive activation of the kinase. However, for most ePKs, genetic manipulations are not required to achieve constant activity, because these enzymes are capable of autoactivating in a mechanism that probably involves dimerization, which enforces a 'prone-to-autophosphorylate' conformation [69-71]. The rate of this spontaneous autophosphorylation is different in each ePK, but in the majority of cases it is sufficient to give rise to a significant activity [69]. In Erks, autoactivation is extremely inefficient and almost non-measurable [72,73]. Erks are fully dependent, therefore, on MAP2Ks for activation loop phosphorylation and induction of catalysis. The lack of autophosphorylation/autoactivation capability in Erk molecules makes overexpression a non-useful experimental approach for studying their biological and pathological effects. As the overall structure of the kinase domain of Erk is very similar to that of the other eEPKs (Figure 1), the explanation for the 
lack of autophosphorylation and basal catalytic activity of Erks is not trivial [69]. Not only that Erks are incapable of autophosphorylation as opposed to most ePKs, several other structural features also distinguish them from common ePKs. For example, although, like most ePKs, Erks seem to reside in equilibrium between two conformations (termed $L$ and $R ;$ [74]) these conformations differ from the classical active and inactive conformations of ePKs. For example, no conformational change in the DFG motif (from 'out' to 'in') is apparent in the structure.

The differences in the biochemical properties between MAPKs and other ePKs may be associated with structural motifs, not part of the kinase domain, which are not present in other ePKs. Two such prominent motifs are the MAPK insert and the C-terminal extension, which includes a domain termed the L16 helix (Figure 1B). However, a bioinformatics-based evolutionary study suggests that the inability to autophosphorylate and the dependence on MEK may stem from minor structural differences, and not necessarily involving the MAPK insert, or the C-terminal extension [75]. This study reconstituted an inferred common ancestor of Erk1, Erk2 and Erk5 that is able to autophosphorylate and an ancestor of Erk1 and Erk2 that cannot. Analysis of the two ancestors suggested that a single amino acid deletion in the linker loop connecting the $\alpha \mathrm{C}$-helix and the $\beta 3$ strand (position 74 in modern Erk1) and a mutation in the gatekeeper residue (Gln122 in modern Erk1) account for the loss of autophosphorylation and dependence of modern Erk on its upstream activator. Indeed, inserting these two modifications into modern Erk1 was sufficient to generate Erk1 molecules that, when tested in kinase assays in vitro, showed high autophosphorylation ability and consequently catalytic capabilities similar to those of Mek-phosphorylated Erk1 [75]. This study clearly points at residues and domains that could be manipulated in an effort to generate intrinsically active, Mek-independent, Erks. These same residues were identified, in fact, as candidates for mutagenesis by other approaches as well [76].

Other unique Erk domains are the substrate binding motifs. Erks possess two distinct sites through which substrates, activators and deactivators can bind. The first is the common docking (CD) site, which is located about $10 \AA$ from the active site of Erk2 (Figure 1B) and is composed of amino acids such as Asp316 and Asp319. The second docking site of Erk2 is the hydrophobic DEF pocket, which is composed of residues Met197, Leu198, Tyr231, Leu232, Leu235 and Tyr261, and exists only in dually phosphorylated Erk adjacent to the catalytic site (Figure 1C). Important mutations, discussed here, occurred in these two domains $[73,77,78]$.

1.1.4. ERK Molecules Are Highly Active in Most Cancers, but Oncogenic Mutations in ERK Themselves Are Very Rare

All upstream components of the Erk signaling cascade are frequently mutated in cancer [79], and it is believed, therefore, that Erks are abnormally overactive in essentially all cancer cases [80]. Accordingly, the Ras-Raf-MEK-Erk cascade has become a major target for anti-cancer therapy [81]. Oncogenic mutations or other genetic alterations (e.g., gene amplification) have been found in RTKs, Ras, Raf and MEKs, but no activating mutations or genetic alterations in Erk molecules themselves have been reported as oncogenic in tumor viruses or in patients. However, as the only known substrates of Rafs are the MEKs, and the only known substrates of MEKs are the Erks, it implies that the biological and pathological/oncogenic effects of the pathway are mediated exclusively via the Erk proteins (note some reports on deviations from the linearity of the RTK-Ras-Raf-MEK-Erk tier: [41,49-51], reviewed in [17]). It is not clear, therefore, why mutations in Erks are rarely found in cancer patients. This situation could be taken as another indication for the unusual tight regulation bestowed on Erks by the specific structural motifs, immunizing them against mutations that render them spontaneously active and oncogenic. Indeed, although some mutations that render Erks intrinsically active and even oncogenic (one mutation) have been discovered in the laboratory, they do not activate Erk to the maximal levels possible (that of Mek-activated Erk), and their oncogenic effect is markedly weaker than that of active, oncogenic, Ras or Raf $[82,83]$.

Nevertheless, mutations in ERKs have been identified in a small number of patients (Table 1) and at least one of those, E320K in ERK2, seems to appear in a few dozen patients suffering from cervical 
and head and neck carcinoma (Table 1B). Perhaps activating mutations in ERKs are not fully oncogenic and do not have a causative effect, but may promote the disease.

\subsubsection{Erk Inhibitors Have Been Recently Developed}

Not only that Erks are obvious targets for anti-cancer therapy because they are the downstream components of the RTK-Ras-Raf-MEK pathway, in the majority of cases of tumors resistant to EGFR, B-Raf and MEK inhibitors, re-activation of Erk is observed [84]. These findings reinforce the need for direct Erk inhibitors. Specific inhibition of Erks should also be a powerful tool for research. For unknown reasons, developing pharmacological Erk inhibitors has lagged behind the development of inhibitors against the other MAP kinases, JNK and p38 and has required unusual efforts. Morris et al., for example, screened approximately five million compounds and performed multiple improvement steps in order to discover SCH772984, a small molecule that inhibits both Erk isoforms with an $\mathrm{IC}_{50}$ at the nano-molar range [85]. Further development of this inhibitor provided an orally administered analog, MK-8353 [86], which is being tested in phase-I clinical trials. Yet another potent Erk inhibitor is BVD-523 (Ulixertinib), a selective and reversible Erk1 and Erk2 ATP competitive molecule [87], which is currently in phase II clinical trials. GDC-0994 (Ravoxertinib) [88], a pyrazolylpyrrole-based inhibitor that was optimized specifically towards Erk by using structure-guided methods [89], is also undergoing clinical testing. Additional compounds that exhibit selectivity towards Erk are LY3214996 [90], FR180204 [91], VRT-11E [92] and the Erk dimerization inhibitor DEL22379 [93]. For a comprehensive description of Erk inhibitors, see [94].

In parallel to the biochemical and pharmacological characterization of the inhibitors, a large number of mutations that render Erk proteins resistant to them have been reported [95-97] (Table 1).

\section{Identification of Various Mutations in ERKs and Study of Their Properties}

\subsection{Almost All Known Mutations in ERKs Have Been Identified Experimentally}

Unlike the many mutations known in RTKs, Ras, Raf and MEK, mostly identified in tumors, almost all mutations known in ERKs have been identified in laboratory setups. Only a few have been identified in cancer patients, and even for those, it is not clear whether they are associated with the disease. Experimental systems were initially designed for identification of intrinsically active Erks. Later, following the development of Erk inhibitors, genetic screens were developed for identification of mutations that would cause drug resistance. The mutations identified in patients, in screens for drug-resistant molecules and for intrinsically active Erks are summarized in Table 1. It should be noted that numeration of the mutations in ERK1 (in text and in Table 1A) refer to the sequence of the human protein and numeration of mutations in ERK2 (in text and in Table 1B) refer to the sequence of rat protein. Notably, mutations that had been discovered (until 2006) in ERK orthologs in lower organisms were summarized in [98] and will not be discussed here.

\subsection{Mutations Produced on the Basis of Structure-Function Studies}

Original attempts to develop intrinsically active Erk molecules took the conventional approach of trying to mimic the activatory phosphorylation of the activation loop. As no phosphomimetic residue is available for tyrosine, this approach was limited to modifying the Thr of the TEY motif and turned out to be ineffective $[99,100]$. In fact, not only was changing this Thr to Glu unsuccessful, but the resulting Erk2 ${ }^{\mathrm{T} 183 \mathrm{E}}$ enzyme showed lower activity than Erk2 ${ }^{\mathrm{WT}}$, even when phosphorylated by MEK $[98,101,102]$. Furthermore, even when mutations that render Erk2 intrinsically active were discovered, combining them with the T183E mutation did not create a more active molecule [103].

Other mutations devised on the basis of structure-function understanding, however, did lead to the development of interesting mutants. For example, mutating the gatekeeper residue of Erk2 resulted in an intrinsically active variant (Q103G and Q103A) [76]. Furthermore, mutating residues that interact with Gln103 provided even more active variants (when tested as purified recombinant 
proteins), primarily Erk2 ${ }^{\mathrm{I} 2 \mathrm{~A}}$ and Erk2 ${ }^{\mathrm{I} 4 \mathrm{~A}}$ [76]. As described above, the gatekeeper residue was also discovered as a site that distinguishes between an inferred ancestor kinase, which is capable of autophosphorylation, and the modern Erk1 and Erk2. Mutating the gatekeeper residue in Erk1 on the basis of comparison to the inferred ancestor (inserting the Q122M mutation) rendered the mutant intrinsically active [75].

The mechanism that renders all these mutants intrinsically active was shown to be the acquisition of an autophosphorylation capability. Namely, the mutations did not impose adoption of the native conformation, but rather unleashed an obstructed autophosphorylation capability and allowed autoactivation. These observations suggest that Erks are similar to most other ePKs that possess the autophosphorylation machinery, but this activity in Erks is not spontaneous. Structural blockers of autophosphorylation in Erks are not known implying that activating mutations could reveal them. An interesting mechanism of action for how substitutions at Gln103 or Ile84 unblock autophosphorylation was suggested by Emrick et al. It was proposed that the mutations induce a pathway of intramolecular interactions leading to flexibility in the activation lip, thereby enabling the phosphoacceptors to reach the catalytic Asp (D147 in Erk2). In the inactive form of Erk2, the intramolecular pathway includes hydrophobic interaction between Leu73, Gln103 or Ile84 and phe166 of the DFG motif, which in turn is linked to L168 through the backbone. The latter forms side-chain interactions with Val186 from the activation lip. Emrick et al. further suggested that T188 forms hydrogen bond with Asp147 and Lys149. These interactions together impede autoactivation by holding the activation lip in a stable conformation. It is therefore not surprising that mutating Leu73, Gln103 or Ile84 would lead to the movement of Phe166 and thus affect the hydrophobic interaction between Leu168 and Val186 and the hydrogen bond between Thr188 and Asp147/Lys149. This would cause an increase in flexibility of the activation loop and eventually autophosphorylation.

Although, when tested in vitro as recombinant proteins, these mutations render the Erk molecules intrinsically active, the significance of the mutants in the gatekeeper and nearby residues in living cells is not clear. While Erk2 ${ }^{\text {I84A }}$ seems to be spontaneously active when expressed in HEK293 cells, it is not spontaneously active in NIH3T3 cells. The equivalent Erk1 mutant, Erk1 $1^{\mathrm{I} 103 \mathrm{~A}}$ is not spontaneously active in either cell line [82]. None of the mutants can oncogenically transform NIH3T3 cells [82], but, intriguingly, several mutations in the gatekeeper of Erk2 (Q103) were identified in screens for drug-resistant Erk2 molecules (Table 1B) and a mutation in I82 (I82T) was found in one cancer patient (Table 1B). Perhaps conversion of these residues to particular amino acids (e.g., Thr), other than those tested so far (i.e., Ala) would render them more active in cells and possibly even oncogenic.

Another mutation that was generated in Erk2 on the basis of structural studies is S151D. This mutation was designed following an alignment of the conserved sequence DLKPSN in MKK1 with the sequence DLKPEN in CAMP-dependent protein kinase. This mutant resulted in a 15-fold enhancement of MKK1 activity [99] and was therefore attempted in Erk2 [103]. Erk2 ${ }^{\text {S151D }}$ manifested MEK-independent activity that was about 15 -fold higher than that of Erk2 ${ }^{\mathrm{WT}}$, but was just $1.5 \%$ of that of MEK-phosphorylated Erk2 ${ }^{\text {WT }}[73,103]$.

\subsection{Only a Few of the Mutations Identified via Genetic Screens in ERK Orthologs of Evolutionarily Low Organisms Are Relevant to Mammalian Erks}

High throughput screens in S. cerevisiae and D. melanogaster, provided a variety of gain-of-function mutants of Erk orthologs in these organisms [73,77,104,105] (Reviewed in [98]). These mutations allowed important insights into the modes of regulation of the given Erk ortholog in each case and to interesting cross talks between yeast MAPK pathways. The relevance of most of those to mammalian Erks is, however, unclear, because some of the mutations occurred in residues that are not conserved in the mammalian enzymes, and of the conserved residues only a handful were tested $[73,103,106]$. Overall, very few of the mutations turned out to be relevant to mammalian Erks, but these are of significant importance. 
For example, insertion of the L73P mutation to Erk2 (equivalent to the L63P mutation in Fus3 [107]) rendered Erk2 intrinsically active, as tested by an in vitro kinase assay with purified recombinant proteins, but to an activity level of approximately $1 \%$ of the activity displayed by Mek-activated Erk2 [103]. Combining L73P with other mutations, such as S151D and D319N, created a more active enzyme [103]. It required a combination of three mutations, L73P+S151D+D319N to create an Erk2 protein with a MEK-independent activity that was 100-fold higher than that of wild type Erk2 in vitro. Notably however, this activity is just about $6 \%$ of the MEK-phosphorylated Erk2 activity [103]. Thus, these mutants are bona fide intrinsically active, but their activity is not very high. Interestingly Leu73 is part of the hydrophobic cluster affected by the "gatekeeper mutations". It seems that mutations in Ser151 also interfere with the contacts of the catalytic base Asp147 with Thr188, resulting in increased activation lip flexibility and activation of the phosphoacceptors Tyr185 and Thr183.

The only Erk mutant that has been shown so far to oncogenically transform cells in cultures, Erk1 ${ }^{\text {R84S }}$, was generated on the basis of a mutation in the yeast ortholog Mpk1/Slt2. The mutation in Mpk1/Slt2, R68S, was identified in a screen that looked for Mpk1 mutants that rescue the phenotype of cells lacking the relevant MEKs [73]. Six Mpk1 mutants were isolated, but only the R68S mutation was found relevant to Erks of higher eukaryotes, including of Drosophila and mammals [73]. Drosophila and mammalian Erks carrying the equivalent mutation (R80S in Drosophila's ERK/Rolled; R84S in mammalian Erk1; R65S in mammalian Erk2) displayed high spontaneous intrinsic catalytic activity $(>30 \%$ of the activity of Mek-activated Erk), independent of Mek activation in in vitro assays [73,82], in cell cultures [82] and in vivo, in transgenic mice and flies [83,108]. Furthermore, Erk1 ${ }^{R 84 S}$ and Rolled ${ }^{\text {R80S }}$ were shown to function as oncogenes, capable of transforming NIH3T3 cells and to give rise to tumors in the fly, respectively $[82,83]$. Erk1 ${ }^{\mathrm{R} 84 \mathrm{~S}}$ was also shown to cause mild cardiac hypertrophy, when expressed as a transgene in the heart of mice [108]. The basic mechanism of action of the R80S/R84S/R65S mutation is similar to that of the other intrinsically active variants described above, namely, it bestowed upon the Rolled and Erk proteins an efficient autophosphorylation capability $[73,82,83]$. The structural basis for this capability is not clear, in part due to the extreme flexibility of Arg65 within the Erk2 structure. In many Erk2 structures it accommodates a different conformation (Figure 2).

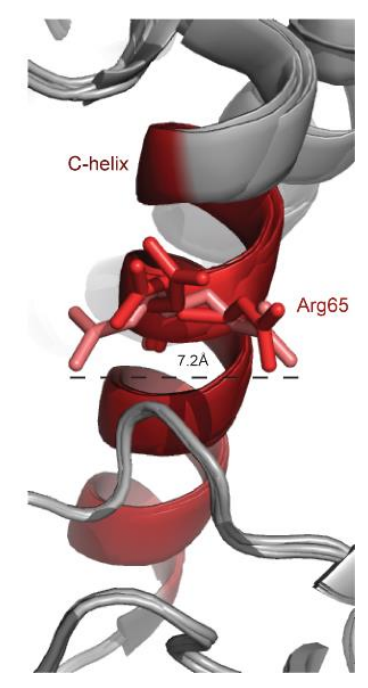

Figure 2. Unusual flexibility of the Arg65 residue at the $\alpha \mathrm{C}$-helix of different Erk2 crystal structures. 5 different crystal structures of Erk2 (PDB 1ERK, 4ERK, 4S31, 4GT3 and 5UMO) were superimposed and a zoom in into the $\alpha \mathrm{C}$-helix (colored in red) is presented. Arg65 is shown in sticks and the distance between the two extreme orientations is calculated.

Arg65 is located at a pivotal position in the $\alpha \mathrm{C}$-helix, the conserved helix within the $\mathrm{N}$ lobe, and interacts with the L16 domain, which is flexible and unstructured in the inactive form. Interestingly, in spite of the different conformation adopted by Arg65 in the various crystal structures of Erk2 (Figure 2), in many of them Arg65 is in association with amino acids of the conserved DFG motif 
(D165, F166, G167). In the structure of Erk2 ${ }^{\text {WT }}$, PDB 1ERK, or 5UMO, Arg65 seems to have two possible conformations so that it forms a hydrogen bond with either the side chain of Asp165 or the backbone of Gly167. In the structure of Erk $2^{\mathrm{R} 65 \mathrm{~S}}$ (determined at a high resolution of $1.48 \AA$ (PDB 4SZ2)) the substituted serine is smaller than arginine and is not in association with the DFG motif. Instead, a new hydrogen bond is formed between Ser65 and Tyr34 of the P-loop (see Figure 8 in reference [82]). In addition, Ser65 stabilizes Thr183 from the activation loop and it is hypothesized that the Asp165 of the DFG motif can interact with ATP. It is noteworthy that in the crystal structure of dually phosphorylated Erk2 (PDB 2ERK), Thr183 interacts with the $\alpha$ C-helix, particularly with Arg65 via a water molecule [109].

Interestingly, similar to the case of Erk2 ${ }^{\mathrm{R} 65 \mathrm{~S}}$, in the crystal structure of the intrinsically active Erk2 ${ }^{\mathrm{I} 4 \mathrm{~A}}$ (PDB code 4S30), a mutant that also autophosphorylates efficiently, the interaction of Arg65 with the DFG is also abolished, although the mutated residue is located in a distance from the $\alpha \mathrm{C}$-helix. As discussed above, the I84A mutation affects a hydrophobic cluster, involving, amongst other residues, Leu73 of the $\alpha \mathrm{C}$-helix, which may in turn divert Arg65. Also, in the Erk2 ${ }^{184 \mathrm{~A}}$ structure in complex with AMP-PNP (PDB 4S34), a shift of Tyr34, causes it to form a hydrogen bond with Thr66 in addition to the Pi-Pi interactions with Tyr62 of the $\alpha \mathrm{C}$-helix observed in Erk2 ${ }^{\mathrm{WT}}$. Tyr34 in Erk2 plays a pivotal role in catalysis and the DFG, especially Asp165, is involved in ATP binding by interacting with the gamma phosphate of ATP. Erk2 bearing mutations in Tyr34 (Y34H/N, in ERK1: Y53H) or Tyr62 (Y62N, in ERK1: Y81C) were shown to acquire resistance to the Erk inhibitors SCH77984 and VRT-11E in both ERK1 and ERK2 in two different screens (see below [95,96]). Finally, the association between Arg65 of Erk2 and the DFG is also abolished in other intrinsically active mutants, including mutations found in the CD site (PDB 6OT6) [110].

It is thus conceivable that the association of Arg65 with the DFG motif is crucial for blocking autophosphorylation activity by acting as a barrier between the ATP binding pocket, DFG motif and the activation loop. Tyr34 and Tyr62 of the P loop, which slightly change their conformation in several active mutants, may also play some role in suppressing spontaneous activity. Notably, mutations in Arg84 of Erk1, equivalent to Arg65 in Erk2, were identified in two cancer patients (Table 1A).

Intriguingly, another gain-of-function mutation, Y268C [73], was identified in the same genetic screen in yeast that provided the R68S mutation in Mpk1/Slt2. It was later shown that Y268A is also a gain-of-function mutation in Mpk1 [78]. Tyr268 is located at the heart of the DEF pocket, and in mammalian Erk2 a mutation in the equivalent Tyr, Y261A, is a partial loss-of-function mutation because Erk2 ${ }^{\mathrm{Y} 261 \mathrm{~A}}$ cannot bind and phosphorylate some of its substrates and cannot execute some of its biological functions $[29,111,112]$. As the mechanism through which Mpk1 ${ }^{\mathrm{Y} 268 \mathrm{~A} / \mathrm{C}}$ function as gain-of-function mutants is unknown, it is also unexplained why the equivalent mutations in Erk2 cause a loss of function [78].

Similar to the mammalian Erks, Rolled, the Erk ortholog in Drosophila melanogaster, is involved in numerous processes in the fly, including the development of the eye $[23,24]$. A screen aimed at isolation of mutants that facilitate proper eye development even in the absence of the ligand that activates the Erk pathway in the eye, identified a mutant fly that was termed sevenmaker. The mutation it carried was found to be D334N in the Drosophila's ERK/Rolled [77]. An equivalent mutation was isolated in another screen in yeast designed for identifying Fus3 molecules that are not inhibited by Hog1 [107], in a large-scale screen performed in mammalian cells for gain-of-function and inhibitor-resistant Erk2 mutants (D319 in Erk2) [95], and in cancer patients. As the sevenmaker mutation occurs at the CD site, its proposed mechanism of action is an elevated resistance to MAPK phosphatases resulting in increased sensitivity to low levels of MEK's activity [106]. This notion was based on the observation that the Erk2 ${ }^{\mathrm{D} 319 \mathrm{~N}}$ protein was less susceptible to inactivation by the MAPK phosphatase CL100 than the Erk2 ${ }^{\mathrm{WT}}$ recombinant protein, while both undergo inactivation in a dose-dependent manner [106]. Inserting this mutation into mammalian Erk2 showed minimal effect on catalytic activity in in vitro experiments and in cell culture $[73,103,106]$. It is not clear how the sevenmaker mutation affects Erk's association with phosphatases, but not with activators and substrates that also utilize the CD site. 
Misiura et al. have shown, quantitatively, that the sevenmaker mutation increases the catalytic activity of Erk by changing the interaction energies. It specifically modifies the enzyme's susceptibility to deactivation by phosphatases, while not affecting the activation process by the MAPK kinase [113]. Nonetheless, the sevenmaker mutation may also affect catalysis per se. Indeed, recombinant Erk2 ${ }^{\mathrm{D} 319 \mathrm{~N}}$ does not manifest unusual catalytic properties, but when combining the D319N mutation with an activating mutation there is a dramatic elevation of catalysis $[73,83,103]$. Since Erk phosphatases do not exist in in vitro assays, the effect of the sevenmaker mutation should be explained by other mechanisms. Probably, in addition to reducing the affinity to phosphatases, the sevenmaker mutation also confers a conformational change that further stabilizes the "prone-to-autophosphorylate" conformation [69] induced by another mutation on the same protein. Supporting the role of the sevenmaker residue not only in substrate binding, but also in activation of catalysis, Molecular Dynamic simulations suggest that stabilization of the active conformation of Erk2, following phosphorylation of Thr183, is associated with disruption of several hydrogen bonding involving Asp334 [114]. Furthermore, in the structure of Erk2 ${ }^{\mathrm{D} 319 \mathrm{~N}}$ the interaction of Arg65 with the DFG is lost, a property of several of the autophosphorylating Erk mutants. Aside from this disruption, the crystal structure of Erk2 ${ }^{\mathrm{D} 319 \mathrm{~N}}$ is, essentially, indistinguishable from that of Erk2 ${ }^{\mathrm{WT}}$ [110].

The sevenmaker site seems to be a hot spot for mutations as it is being re-discovered in different screens. Mutations in Asp319 of Erk2 (D319N, D319V) or mutations in the neighboring residue (E320K and E320V), as well as in Glu79, which associates with D319, (E79K), were identified in a comprehensive screen that searched for gain-of-function and drug-resistant mutations, using A375 cells (see below; [95]). The sevenmaker mutation, itself, D319N, was also reported in four cases of carcinoma (COSMIC ID: D319N in ERK2-COSM98175) and three other patients carried another substitution in the 319 position (Table 1B). Interestingly, the ERK1 sevenmaker site was not found to be mutated in any screen, or in cancer patients.

\subsection{Mutations in ERKs Are Very Rare in Cancer Patients, but Some Are Similar to Those Identified in Laboratory Models}

Although several dozens of mutations in ERK1 and ERK2 have been identified in cancer patients (Table 1) the rate of mutations in ERK in patients is very low and most of the mutations appeared in only one of the samples tested. Mutations in ERK2 were observed in 179 out of 60,712 unique samples, approximately $0.3 \%$. The COSMIC database lists 148 reported ERK1 mutations, out of 47,784 tumor samples tested (also approximately $0.3 \%$ ). It is not clear whether these mutations have any causative effect on the malady. An exception is the E320K mutation, which was observed in 27 patients of squamous cell carcinoma (COSMIC ID: E320K in ERK2-COSM461148). Mutations in Glu320 were also identified in a screen for drug resistant Erk molecules. Notably Glu320 is neighboring Asp319 (the location of the sevenmaker mutation) within the CD site. Just like the sevenmaker mutation, the E320K does not affect the enzyme's intrinsic catalytic properties as tested in vitro with recombinant Erk2 ${ }^{\mathrm{E} 320 \mathrm{~K}}$, and in transient transfections of HEK293 and NIH3T3 cells ([110]; Smorodinsky-Atias and Engelberg, unpublished observation). It may function, therefore, similar to the mutations in Asp319 by reducing the protein association with phosphatases (also supported by Brenan et al. [95]). Yet, unlike D319N, the E320K mutation enforces significant structural changes on the crystal structure of Erk2 and on its biophysical properties. Also, when equivalent mutations were inserted to the Drosophila ERK/Rolled they conferred different properties on the protein, suggesting that D319N and E320K function via different mechanisms $[110,115]$. More mutations of note identified in patients occurred in Glu79 (COSMIC ID: in ERK2-COSM444794), Ser140 (COSMIC ID: in ERK2-COSM3552430) and Pro56 (COSMIC ID: in ERK2-COSM4471756) residues. These substitutions were subsequently discovered as gain-of-function mutations in the screen that tested all possible missense mutations of ERK2 [95] (see below).

Nearly all of the ERK1 mutations recorded in patients, appeared only once. Only 18 mutations occurred in two or three independent samples. Notably, two patients carried the R84H mutation in 
ERK1. As discussed above, another mutation at the same location, R84S, was shown to render Erk1 capable to oncogenically transform cells in culture [82]. This finding calls for testing the oncogenic potential of Erk $1^{\mathrm{R} 84 \mathrm{H}}$ and perhaps of more Erk1 molecules in which Arg84 was substituted.

\subsection{A Large Number of Mutations Can Render Erks Resistant to Pharmacological Inhibitors}

The development of specific inhibitors towards Erks was the impetus for a series of studies that searched for mutations that cause drug resistance. Goetz et al., constructed a library of randomly mutagenized ERK1 and ERK2 cDNAs and induced its expression in A375 melanoma cells (harboring the $\mathrm{BRAF}^{\mathrm{V} 660 \mathrm{E}}$ oncogene) in the presence of either the Erk inhibitor VRT-11E, the MEK inhibitor trametinib, or with a combination of trametinib and the Raf inhibitor dabrafenib [96]. Overall, sequencing the ERK1 or ERK2 molecules in cell populations that survived the treatment identified 33 mutations in ERK1 (in 28 amino acids) and 24 in ERK2 (in 20 amino acids). In a separate screen for A375 colonies resistant to VRT-11E, another five mutations in ERK2 were discovered. All mutations are presented in Table 1. Only five of the mutations that caused resistance to VRT-11E were identified in both isoforms. These were (in ERK1/ERK2 order) Y53H/Y34H/N, G54A/G35S, P75L/P56L, Y81C/Y62N and C82Y/C63Y. A mutation at only one of the residues that caused resistance to the MEK inhibitor was also common in the two isoforms, $\mathrm{Y} 148 \mathrm{H} / \mathrm{Y} 129 \mathrm{~N} / \mathrm{H} / \mathrm{F} / \mathrm{C} / \mathrm{S}$. Importantly, some of the residues that were found mutated in this screen were also reported to be mutated in patients, including Arg84 and Gly186 in Erk1 and Asp319 and Glu320 in Erk2. Another important finding of this study, with significant implications to therapeutic strategies, is that Erk variants that are resistant to RAF/MEK inhibitors are sensitive to Erk inhibition and vice versa [96].

As the mutations were not tested on purified Erk proteins it is not known how they affect the intrinsic biochemical and structural properties of the enzymes leaving the detailed mechanism of the acquired drug resistance open for future studies. It is possible however that mutations identified in the Erk-inhibitor screen interfere with drug binding as they cluster in proximity to the ATP/drug binding pocket. These mutations are located in the glycine-rich loop and the loop between $\beta 3$ and $\alpha \mathrm{C}$-helix (in ERK1: I48N, Y53H, G54A, S74G, P75L. In ERK2: Y34H, G71S, P56L). Mutations residing in the $\alpha$ C-helix itself (ERK1: Y81C, C82Y, ERK2: Y62N, C63Y) may function similarly. ERK1 mutants that were identified in the Raf/MEK inhibitors screen probably function via a different mechanism. They are distributed along the molecule, but seem to cluster in domains important for catalysis and may render the kinase catalytically active. A206V/A187V and S219P/S200P (of ERK1/ERK2), for example, reside in the activation lip, R84H, C82Y and Q90R of Erk1 map to the $\alpha \mathrm{C}$-helix and Y148H of Erk1 and Y129F, D319G and E320K of Erk2 are located in the CD site. Mutations in the sevenmaker residue were already discussed above and probably function by reducing affinity to phosphatases in combination with some effect on catalysis. Another mutation, R84H in Erk1, occurred at the same residue in which the only oncogenic mutation identified so far in Erks, R84S, also occurred [82]. As Erk $1^{R 84 S}$ was shown to be intrinsically catalytically active and spontaneously active when expressed in culture cells and in transgenic mice $[82,108]$, it could be that Erk1 $1^{\mathrm{R} 84 \mathrm{H}}$ also acquired similar properties and is independent of upstream activation, making it resistant to Raf and MEK inhibitors. Indeed, kinase assays using the various mutants, expressed in and immunoprecipitated from A375 cells, showed that the mutants maintained activity in the presence of either VRT-11E or SCH772984, another Erk inhibitor [96]. On the basis of the immunoprecipitation kinase assay it could be suggested that the mutants arising from the screen in the presence of RAF/MEK inhibitors acquired the capability to maintain sufficient kinase activity, even under these conditions, thereby rescuing the transformed cells from the inhibitors. As some of these mutations are found in patients, this conclusion is therapeutically relevant and may suggest that RAF and MEK inhibitors should be contraindicated for patients that harbor these mutants in the tumor.

Intriguingly, the mechanisms of action of the various mutants in vivo may be different for Erk1 and Erk2 as some of the mutations seem to be isoform-specific. Mutations in $\alpha \mathrm{C}$-helix were found only in ERK1 (C82Y, R84H and Q90R), while mutations in the CD site, such as D319G and E320K were 
only found in Erk2. Although the differences in the occurrence of the mutations could be a result of an unsaturated screen, the notion that the mutations are isoform-specific is supported by the situations observed in patients. The E320K mutation of Erk2 was found in 27 cancer patients while the equivalent mutation in Erk1 was not reported. Similarly, the sevenmaker site of Erk2, D319, which was mutated in seven patients, was not found so far to be mutated in ERK1 in patients. In line with these observations Goetz et al. inserted the equivalents of the D319G and E320K mutations into ERK1, and observed that the resulting proteins, Erk1 $1^{\mathrm{D} 338 \mathrm{~N}}$ and Erk1 ${ }^{\mathrm{E} 339 \mathrm{~K}}$ were not resistant to inhibitors, at least in the cell assay [96]. It is reasonable to conclude that different mutations may render Erk1 and Erk2 resistant to drugs. Given that the biological functions of the two isoforms is almost fully redundant [36] and that the inhibitors affect both isoforms similarly in vitro, it is currently difficult to explain the dichotomy in the mutations that cause resistance of each isoform.

A mutation that renders Erks resistant to SCH772984 was identified when cells of the colorectal cancer cell line HCT-116 (harboring a mutated KRAS) were serially passaged in the presence of increasing concentration of the inhibitor for 4 months [97]. Sequencing the ERK1/2 genes isolated from resistant clones, revealed a reoccurring point mutation, G186D, in ERK1 [97]. Gly186 resides in the DFG motif of the activation segment. The mutant displayed a several-fold reduction in binding affinity to the inhibitor, compared to the wild-type protein. Crystal structure of SCH772984-associated Erk2 suggests that this reduction is a direct result of a steric clash imposed by the aspartic acid in the active site, which destabilized the binding of the inhibitor. The same Erk1 ${ }^{\mathrm{G} 186 \mathrm{D}}$ mutant did not provide resistance to another ATP-competitive Erk inhibitor (VRT-11E), an observation that is explained by the structural difference between the two inhibitors, significantly altering the interactions with the binding pocket, predominantly the distance of the molecule from the new aspartic acid [97]. Notably, the orthologous residue in Erk2, Gly167 was found mutated to Asp in a screen for Erk2 mutants that are resistant to SCH772984 and VRT-11E in A375 cells. In this screen Gly167 was mutated to many other residues, but only the Erk2 ${ }^{\mathrm{G} 167 \mathrm{D}}$ was selected as rendering the kinase drug-resistance [95]. The interference of Asp at position 169 for drug binding seems very particular.

Erk2 $2^{\mathrm{G} 167 \mathrm{D}}$ was in fact isolated in a large-scale screen that led to the discovery of many more mutants. In this effort, Brenan et al. employed saturation mutagenesis and were able to screen a library of 6810 variants of ERK2, out of 6821 possible, each carrying a point mutation. They searched for gain-of-function, loss-of-function, as well as for drug-resistant mutants. The ERK2 mutants library was introduced into A375 cells and inducibly expressed, under the premise that cells expressing gain-of-function mutants will proliferate slower, while cells expressing loss-of-function mutants will proliferate faster, than cells expressing Erk $2^{\mathrm{WT}}$ [95]. The relative abundance of Erk2 molecules that were expressed in the cells after $96 \mathrm{~h}$ was determined by parallel sequencing.

Indeed, mutants considered to be carrying GOF mutations on the basis of previous screens or appearance in tumors, such as Glu320 and Asp319, were depleted from the proliferative culture. Mutations in an additional 19 residues caused the Erk2 molecules to be depleted to a degree equivalent to or greater than that of Erk2 ${ }^{\text {Glu320 }}$ and Erk2 ${ }^{\text {Asp319 }}$. The 19 residues were Glu79, Gly83, Gly8, Leu333, Pro56, Val47, Ser358, Val16, Pro354, Arg13, Glu320, Glu58, Ala3, Ala350, Ala7, Ser140, Thr24, Gln15, Asp319, Gly20 and Phe346 (from the most depleted to the least). Many of the mutants considered to have acquired gain-of-function mutations were shown to be catalytically active when immunoprecipitated from cells treated with the RAF inhibitor trametinib. As the mutants were not tested as recombinant purified proteins it is not clear whether they possess any unusual catalytic properties, and, specifically, if they are intrinsically active.

The mutants library was also inducibly expressed in A375 cells exposed to sublethal doses of VRT-11E, or SCH772984 in order to discover Erk2 mutants that are resistant to these inhibitors. The relative enrichment of the Erk2 mutants was quantified after 12 days of exposure to inhibitors. The rationale in this experiment was that cells harboring an Erk2 mutant insensitive to an inhibitor would allow proliferation in its presence. Mutations in 12 residues rendered Erk2 resistant to VRT-11E (Arg13, Ile29, Gly30, Ala33, Met36, Val37, Arg65, Gln95, Met96, Asp98, Thr108, Leu154), mutations in 
9 residues confer resistance to SCH772984 (Glu31, Tyr41, Val47, Lys53, Glu68, Leu67, Ile101, Asp122, Asp125) and mutations in 18 residues render Erk2 resistant to both inhibitors (Tyr34, Gly35, Cys38, Asp42, Val49, Ile54, Ser55, Pro56, Phe57, Gln60, Tyr62, Cys63, Thr66, Glu69, Leu73, Gln103, Gly167, Ile345). Curiously, half of the mutations that cause resistant to VRT-11E occurred in residues that make direct contacts with the inhibitor, but none of the mutants that confer specific resistance to SCH772984 are mutated in residues that contact the inhibitor. Projection of the mutations identified in this study into cancer associated mutations showed that within the top 20 residues harboring GOF mutations, five were reported to be mutated in patients (E320K/V, D319N/V, E79K, P56L and S140L). Erk2 mutants carrying any of these GOF mutations were found to rescue A375 cells from the anti-proliferative effect of Raf/MEK inhibitors, but only one, Erk2 ${ }^{\mathrm{P} 56 \mathrm{~L} / \mathrm{G}}$ was able to rescue the cells from SCH772984. All GOF mutations clustered within the CD site, whereas LOF mutations altered the DEF pocket. The LOF mutations in the DEF pocket seem dominant since when combined, on a single Erk2 molecule, with a GOF mutation in the CD site the resulting protein was not able to rescue cells and promote the downstream signaling. It seems that the mechanism of action of the GOF mutants residing in the $\mathrm{CD}$ site is similar to that of the sevenmaker mutation. This hypothesis was validated by co-expressing Erk2 mutants with BRAF ${ }^{\mathrm{V} 600 \mathrm{E}}$ and dual specificity phosphatase (DUSP) in 293T cells and monitoring Erk2 phosphorylation. Erk2 molecules mutated at the CD site (E320K/V; D319N/V E79K) exhibited sustained phosphorylation levels relative to Erk2 ${ }^{\mathrm{WT}}$ [95]. It is worth noting that the GOF mutant P56L showed a reduced level of phosphorylation, suggesting that constant phosphorylation in the presence of DUSP is not a general property of all GOF mutations. Most mutants identified in this comprehensive study await further biochemical, structural and pharmacological analysis.

\section{Discussion}

Table 1 lists an impressive number of mutations in Erk1 and Erk2, many of them discovered in screens for drug-resistant mutants. The mutations that confer drug resistance outscore the very few mutations that render Erks intrinsically active and the single mutant that was shown so far to possess oncogenic properties. The mutations causing drug-resistant occur throughout the protein so that various mechanisms are involved. It is not clear whether the list in Table 1 reflects all relevant mutations possible in Erks. Indeed, most of the screens that provided the currently known mutations were high throughput, but they were performed in a particular context of a given cell line, or against specific pharmacological inhibitors. Also, it is not clear if these screens themselves were saturated. It is a plausible assumption therefore that further screening, particularly in novel experimental setups, would result in yet unidentified mutations. Also, once Erk inhibitors reach the clinic, patients may develop resistance by acquiring mutations other than those discovered in the laboratory. As the research of Erk mutations is relatively young many properties of the known mutation are still elusive. For most of the mutations that cause resistant to inhibitors the mechanism of action is not known and for many of them even the effects on Erks' conformation and catalytic properties are yet to be revealed. Finally, it may take a long time to reveal the role (if any) of the rare mutations identified in cancer patients, in disease etiology. Most mutations that were identified in a single patient, may be bystanders with no role in the disease, while others may contribute to disease development or even disease onset. Prime suspects for a causative role are the R84H mutation in Erk1, because an equivalent mutation, R84S, was shown to be oncogenic in cells in culture, and E320K in Erk2 that is found in patients in a higher rate than all other mutations.

\subsection{Mutations Identified in Erks Could Not Have Been Predicted by Structural or Mechanistic Analysis}

It is not currently possible to predict the location and type of more Erk mutations. Although Erk proteins have been the subject of comprehensive structural studies, including via X-ray crystallography, NMR and HX-MS approaches, and although critical features of their structure-function properties have been revealed, it is not clear how to translate this knowledge into predicting specific mutations that will modify Erks' biochemical or pharmacological properties or biological functions. Also, modifying 
Erks' biological effects requires understanding of additional mechanisms, responsible for sub-cellular localization and interaction with partners and scaffold proteins. Some of these mechanisms could be isoform-specific and, consequently, mutations that affect these processes may be different in Erk1 and Erk2, similar to the case of the CD site mutations that seem relevant only to Erk2 and mutation in the $\alpha \mathrm{C}$-helix, which are more relevant to Erk1. As a result of our inability to translate structural and mechanistic knowledge into mutation design, most mutations identified so far were discovered via unbiased screens, and even following their isolation, their mechanism of action is not understood.

\subsection{Mutations Identified in Erks Disclose 3 Hotspots for Mutagenesis, Perhaps Reflecting Some Prevailing Mechanisms}

Although mutations discussed in this review are spread along the Erk molecules, some hotspots re-appear in several laboratory screens as well as in cancer patients. The sevenmaker residue and its neighbor at the CD site, Glu320, are prominent examples, relevant for Erk2. $\alpha \mathrm{C}$-helix, mainly Arg84/Arg65 (in ERK1/ERK2), is another hotspot, which seems relevant primarily to Erk1. Yet another important residue is the gatekeeper that was discovered as a target for mutagenesis by studying the evolution of Erks and by structural approaches. Mutations that occur in the CD site affect protein-protein interactions, primarily with phosphatases, while mutations in the gatekeeper, in the residues that are in proximity to it and in residues of the $\alpha \mathrm{C}$-helix, significantly increase the commonly negligible intrinsic catalytic activity of the kinases. All mutations that caused elevation of the basal catalytic activity did so via identical mechanism, increasing the autocatalytic capability of the proteins. So far, no mutation was discovered that allows Erks to bypass the requirement of activation loop phosphorylation and enforces by itself adoption of the active conformation. Rather, the mutations discovered so far caused the Erk molecule to acquire a 'prone to autophosphorylate' conformation. Erks may be immune against mutations that induce the active conformation by themselves.

\section{Conclusions}

\section{Clinical and Biochemical Lessons from the Erk Mutant}

Most of the mutations described in this review seem to be directly relevant to understanding cancer etiology and patients response to drugs. An important lesson is that Erks, at least Erk1, could become oncogenic. This finding strongly suggests that the oncogenicity of the RTK-Ras-Raf-MEK pathway is mediated primarily via Erks, reinforcing the effort to inhibit Erk as a powerful anti-cancer approach. The analysis of the mutants may further suggest that isoform-specific inhibitors should be developed with higher priority to Erk1-specific inhibitors. This would be a difficult challenge for drug developers. The mutations that cause drug resistance could be already taken into consideration when a therapeutic strategy is planned. Namely, drugs should be applied according to the mutation that appears in the tumor. This requires deep understanding of the effect of each of the many mutations discovered so far (Table 1) on Erks' biochemistry, biology and pathology. 
Table 1. (A) Mutations identified in ERK1 (MAPK3; mutations numeration is according to the sequence of the human ERK1). (B) Mutations identified in ERK2 (MAPK1) (numeration of the mutants is according to the sequence of rat ERK2).

\begin{tabular}{|c|c|c|}
\hline Mutation & Mode of Identification & Reference \\
\hline \multicolumn{3}{|c|}{$(\mathbf{A})$} \\
\hline A6_Q7insA & In cancer patients & COSMIC, cBioPortal \\
\hline A6dup & In cancer patients & COSMIC \\
\hline Q7R & In cancer patients & COSMIC \\
\hline Q7H & In cancer patients & COSMIC, cBioPortal \\
\hline G10R & In cancer patients & COSMIC \\
\hline G11V & In cancer patients & COSMIC \\
\hline G11_Splice & In cancer patients & COSMIC, cBioPortal \\
\hline G12E & In cancer patients & COSMIC \\
\hline E13K & In cancer patients & COSMIC, cBioPortal \\
\hline E13* & In cancer patients & COSMIC, cBioPortal \\
\hline R15G & In cancer patients & COSMIC, cBioPortal \\
\hline R16I & In cancer patients & COSMIC, cBioPortal \\
\hline E18Q & In cancer patients & COSMIC, cBioPortal, TumorPortal \\
\hline V20V & In cancer patients & COSMIC \\
\hline G23G & In cancer patients, and in a screen for mutants resistant to Erk inhibitors & COSMIC, [96] \\
\hline $\mathrm{V} 24 \mathrm{~F}$ & In cancer patients & COSMIC, cBioPortal \\
\hline V24S & In cancer patients & cBioPortal \\
\hline $\mathrm{V} 24 \mathrm{fs} * 8$ & In cancer patients & cBioPortal \\
\hline P25S & In cancer patients & COSMIC, cBioPortal \\
\hline $\mathrm{E} 27 \mathrm{fs} * 35$ & In cancer patients & COSMIC, cBioPortal \\
\hline E27G & In cancer patients & COSMIC \\
\hline M30I & In cancer patients & COSMIC, cBioPortal \\
\hline
\end{tabular}


Table 1. Cont.

\begin{tabular}{|c|c|c|}
\hline Mutation & Mode of Identification & Reference \\
\hline G33W & In cancer patients & cBioPortal \\
\hline P35S & In cancer patients & COSMIC \\
\hline D37D & In cancer patients & COSMIC, TumorPortal \\
\hline Q46H & In cancer patients & cBioPortal \\
\hline $\mathrm{I} 48 \mathrm{~N}$ & In a screen for mutants resistant to VRT-11E & [96] \\
\hline G51S & In a screen for mutants resistant to RAF/MEK inhibitors & [96] \\
\hline A52P & In cancer patients & COSMIC, cBioPortal \\
\hline Y53Y & In cancer patients & COSMIC \\
\hline $\mathrm{Y} 53 \mathrm{H}$ & In a screen for mutants resistant to VRT-11E and SCH772984 & [96] \\
\hline Y53C & In a screen for mutants resistant to Erk inhibitors & [96] \\
\hline G54S & In a screen for mutants resistant to VRT-11E and SCH772984 & [96] \\
\hline G54C & In cancer patients & cBioPortal \\
\hline G54A & In a screen for mutants resistant to Erk inhibitors & [96] \\
\hline S57G & In a screen for mutants resistant to RAF/MEK inhibitors & [96] \\
\hline X57_splice & In cancer patients & cBioPortal \\
\hline S58L & In cancer patients & cBioPortal, TumorPortal \\
\hline Y60C & In a screen for mutants resistant to Erk inhibitors & [96] \\
\hline D61E & In cancer patients & COSMIC, cBioPortal \\
\hline H62_S74>R & In cancer patients & COSMIC, cBioPortal \\
\hline $\mathrm{V} 63 \mathrm{M}$ & In cancer patients & COSMIC \\
\hline $\mathrm{R} 64 \mathrm{C}$ & In cancer patients & COSMIC, cBioPortal \\
\hline $\mathrm{R} 64 \mathrm{~L}$ & In cancer patients & cBioPortal \\
\hline K65R & In a screen for mutants resistant to Erk and RAF/MEK inhibitors & [96] \\
\hline $\mathrm{R} 67 \mathrm{C}$ & In cancer patients & COSMIC \\
\hline
\end{tabular}


Table 1. Cont

\begin{tabular}{|c|c|c|}
\hline Mutation & Mode of Identification & Reference \\
\hline V68L & In cancer patients & cBioPortal \\
\hline $\mathrm{K} 72 \mathrm{~N}$ & In cancer patients & COSMIC, cBioPortal \\
\hline K72R & In a screen for mutants resistant to Erk inhibitors & [96] \\
\hline I73S & In a screen for mutants resistant to Erk inhibitors & [96] \\
\hline $\mathrm{I} 73 \mathrm{M}$ & In Cancer patients & COSMIC, cBioPortal \\
\hline S74G & In a screen for mutants resistant to VRT-11E & [96] \\
\hline Insertion $74 \mathrm{~N}$ & An activating mutation. On the basis of inferred ancestor & [75] \\
\hline P75P & In Cancer patients & TumorPortal \\
\hline P75L & In a screen for mutants resistant to VRT-11E and SCH772984 & [96] \\
\hline P75S & In a screen for mutants resistant to VRT-11E and SCH772984 & [96] \\
\hline E77E & In cancer patients, and in a screen for mutants resistant to Erk inhibitors & COSMIC, cBioPortal, TumorPortal, [96. \\
\hline $\mathrm{Q} 79 \mathrm{H}$ & In cancer patients & cBioPortal \\
\hline Q79* & In cancer patients & COSMIC, cBioPortal, TumorPortal \\
\hline Y81C & In a screen for mutants resistant to VRT-11E & [96] \\
\hline $\mathrm{C} 82 \mathrm{Y}$ & In cancer patients & cBioPortal, [96] \\
\hline $\mathrm{R} 84 \mathrm{H}$ & $\begin{array}{l}\text { In cancer patients, and in a screen for mutants resistant to trametinib } \\
\text { and dabrafenib }\end{array}$ & COSMIC, cBioPortal,[96] \\
\hline R84S & $\begin{array}{l}\text { In a screen for mutants resistant to RAF/MEK inhibitors, and in a screen for } \\
\text { MEK-independent mutants }\end{array}$ & {$[73,96]$} \\
\hline $\mathrm{T} 85 \mathrm{~T}$ & In cancer patients & COSMIC \\
\hline L86L & In cancer patients & COSMIC \\
\hline L86R & In a screen for mutants resistant to RAF/MEK inhibitors & [96] \\
\hline L86P & In a screen for mutants resistant to RAF/MEK inhibitors & [96] \\
\hline R87W & In cancer patients & COSMIC, cBioPortal \\
\hline
\end{tabular}


Table 1. Cont

\begin{tabular}{|c|c|c|}
\hline Mutation & Mode of Identification & Reference \\
\hline Q90R & In a screen for mutants resistant to trametinib and dabrafenib & [96] \\
\hline $\mathrm{R} 94 \mathrm{C}$ & In cancer patients & COSMIC, cBioPortal, TumorPortal \\
\hline $\mathrm{R} 96 \mathrm{H}$ & In cancer patients & COSMIC, cBioPortal \\
\hline R96C & In cancer patients & cBioPortal \\
\hline R96R & In cancer patients & COSMIC \\
\hline $\mathrm{H} 97 \mathrm{H}$ & In cancer patients & COSMIC \\
\hline E98K & In cancer patients & COSMIC, cBioPortal \\
\hline E98D & In cancer patients & COSMIC, cBioPortal \\
\hline I101I & In cancer patients & COSMIC \\
\hline G102D & In cancer patients & COSMIC, cBioPortal \\
\hline I103A & On the basis of structural considerations & {$[76]$} \\
\hline R104Q & In cancer patients & cBioPortal \\
\hline L107L & In cancer patients & TumorPortal \\
\hline R108W & In cancer patients & COSMIC, cBioPortal \\
\hline A109P & In cancer patients & COSMIC \\
\hline Q122M & An activating mutation. On the basis of inferred ancestor & [75] \\
\hline L124L & In cancer patients & COSMIC \\
\hline E126* & In cancer patients & COSMIC, cBioPortal, TumorPortal \\
\hline $\mathrm{S} 135 \mathrm{~N}$ & In a screen for mutants resistant to RAF/MEK inhibitors & [96] \\
\hline Q136fs*4 & In cancer patients & cBioPortal \\
\hline Q136* & In cancer patients & cBioPortal \\
\hline Q136H & In cancer patients & cBioPortal \\
\hline L138L & In cancer patients & COSMIC \\
\hline
\end{tabular}


Table 1. Cont.

\begin{tabular}{|c|c|c|}
\hline Mutation & Mode of Identification & Reference \\
\hline $\mathrm{H} 142 \mathrm{H}$ & In cancer patients & COSMIC \\
\hline Y145* & In cancer patients & COSMIC \\
\hline $\mathrm{Y} 148 \mathrm{H}$ & In a screen for mutants resistant to trametinib and dabrafenib & [96] \\
\hline Y148C & In cancer patients & COSMIC, cBioPortal \\
\hline R152L & In cancer patients & TumorPortal \\
\hline R152W & In cancer patients & COSMIC, cBioPortal, TumorPortal \\
\hline G153S & In cancer patients & COSMIC, cBioPortal \\
\hline G153G & In cancer patients & COSMIC \\
\hline H158L & In cancer patients & COSMIC \\
\hline S159S & In cancer patients & COSMIC, TumorPortal \\
\hline A160T & In cancer patients & COSMIC, cBioPortal \\
\hline A160A & In cancer patients & COSMIC \\
\hline L163L & In cancer patients & COSMIC \\
\hline R165L & In cancer patients & COSMIC \\
\hline P169L & In cancer patients & cBioPortal \\
\hline N171D & In cancer patients & COSMIC, cBioPortal \\
\hline T177I & In cancer patients & COSMIC, cBioPortal \\
\hline C178R & In cancer patients & COSMIC \\
\hline $\mathrm{C} 178 \mathrm{Y}$ & In cancer patients & COSMIC \\
\hline $\mathrm{C} 178 \mathrm{C}$ & In cancer patients & COSMIC \\
\hline D179N & In cancer patients & COSMIC \\
\hline $\mathrm{I} 182 \mathrm{~N}$ & In cancer patients & cBioPortal \\
\hline I182-splice & In cancer patients & TumorPortal \\
\hline F185F & In cancer patients & COSMIC \\
\hline
\end{tabular}


Table 1. Cont

\begin{tabular}{|c|c|c|}
\hline Mutation & Mode of Identification & Reference \\
\hline F185I & In cancer patients & cBioPortal \\
\hline G186R & In cancer patients & COSMIC, cBioPortal, TumorPortal \\
\hline G186D & In a screen for mutants resistant to VRT-1E and SCH772984 & {$[96,97]$} \\
\hline R189W & In cancer patients & COSMIC, cBioPortal \\
\hline R189Q & In cancer patients & COSMIC \\
\hline R189R & In cancer patients & COSMIC \\
\hline I190T & In cancer patients & COSMIC, cBioPortal \\
\hline P193T & In cancer patients & COSMIC, cBioPortal \\
\hline $\mathrm{P} 193 \mathrm{H}$ & In cancer patients & COSMIC, cBioPortal \\
\hline P193S & In cancer patients & cBioPortal, TumorPortal \\
\hline E194Q & In cancer patients & COSMIC, cBioPortal \\
\hline T198T & In cancer patients & COSMIC \\
\hline G199D & In cancer patients & COSMIC, cBioPortal \\
\hline T202M & In cancer patients & COSMIC, cBioPortal \\
\hline E203K & In cancer patients & cBioPortal \\
\hline A206V & In a screen for mutants resistant to trametinib and dabrafenib & [96] \\
\hline T207T & In cancer patients & COSMIC, TumorPortal \\
\hline R211Q & In cancer patients & COSMIC, cBioPortal \\
\hline $\mathrm{R} 211 \mathrm{P}$ & In cancer patients & COSMIC, cBioPortal \\
\hline $\mathrm{R} 211 \mathrm{~W}$ & In cancer patients & cBioPortal \\
\hline E214D & In cancer patients & cBioPortal \\
\hline M216I & In cancer patients, and in a screen for mutants resistant to RAF/MEK inhibitors & COSMIC, cBioPortal,[96] \\
\hline $\mathrm{N} 218 \mathrm{~N}$ & In cancer patients & COSMIC \\
\hline
\end{tabular}


Table 1. Cont

\begin{tabular}{|c|c|c|}
\hline Mutation & Mode of Identification & Reference \\
\hline S219F & In cancer patients & COSMIC, cBioPortal \\
\hline S219P & In a screen for mutants resistant to trametinib and dabrafenib & [96] \\
\hline X221_splice & In cancer patients & cBioPortal \\
\hline $\mathrm{D} 227 \mathrm{~N}$ & In cancer patients & cBioPortal \\
\hline V231L & In cancer patients & COSMIC \\
\hline $\mathrm{A} 236 \mathrm{~T}$ & In cancer patients & COSMIC \\
\hline S240C & In cancer patients & COSMIC \\
\hline $\mathrm{R} 242 \mathrm{R}$ & In cancer patients & TumorPortal \\
\hline L251L & In cancer patients & COSMIC \\
\hline Q253P & In cancer patients & COSMIC, cBioPortal \\
\hline $\mathrm{I} 257 \mathrm{~V}$ & In cancer patients & COSMIC, cBioPortal \\
\hline $\mathrm{I} 260 \mathrm{H}$ & In cancer patients & COSMIC, cBioPortal \\
\hline Q266* & In cancer patients & COSMIC, cBioPortal \\
\hline L269P & In cancer patients & COSMIC, cBioPortal, TumorPortal \\
\hline I273M & In Cancer patients & cBioPortal \\
\hline $\mathrm{R} 278^{*}$ & In cancer patients, and in a screen for mutants resistant to RAF/MEK inhibitors & cBioPortal, [96] \\
\hline L281I & In cancer patients & cBioPortal \\
\hline V290A & In cancer patients & COSMIC, cBioPortal, TumorPortal \\
\hline F296F & In cancer patients & COSMIC \\
\hline D300E & In cancer patients & COSMIC, cBioPortal \\
\hline $\mathrm{A} 303 \mathrm{~V}$ & In cancer patients, and in a screen for mutants resistant to RAF/MEK inhibitors & COSMIC, cBioPortal, [96] \\
\hline L304P & In Cancer patients & cBioPortal \\
\hline L306L & In Cancer patients, and in a screen for mutants resistant to Erk inhibitors & cBioPortal, [96] \\
\hline $\mathrm{T} 312 \mathrm{~S}$ & In cancer patients & cBioPortal \\
\hline
\end{tabular}


Table 1. Cont.

\begin{tabular}{|c|c|c|}
\hline Mutation & Mode of Identification & Reference \\
\hline N314I & In cancer patients & COSMIC, cBioPortal, TumorPortal \\
\hline $\mathrm{N} 314 \mathrm{~N}$ & In cancer patients & COSMIC \\
\hline P315H & In cancer patients & COSMIC \\
\hline R318W & In cancer patients & COSMIC, cBioPortal \\
\hline R318R & In cancer patients & COSMIC \\
\hline $\mathrm{V} 321 \mathrm{fs} * 40$ & In cancer patients & cBioPortal \\
\hline V321W & In cancer patients & cBioPortal \\
\hline P328L & In cancer patients & COSMIC, cBioPortal \\
\hline E331E & In cancer patients & COSMIC \\
\hline Q332H & In cancer patients & COSMIC \\
\hline D335N & In cancer patients & COSMIC, cBioPortal \\
\hline P336Q & In cancer patients & COSMIC \\
\hline T337T & In cancer patients & COSMIC \\
\hline E339V & In cancer patients & COSMIC, cBioPortal \\
\hline E343K & In cancer patients & cBioPortal \\
\hline P345T & In cancer patients & COSMIC \\
\hline F346I & In cancer patients & COSMIC, cBioPortal \\
\hline F346F & In cancer patients & COSMIC \\
\hline F348F & In cancer patients & COSMIC \\
\hline A349T & In cancer patients & cBioPortal \\
\hline R359W & In cancer patients & COSMIC, cBioPortal, TumorPortal \\
\hline R359Q & In cancer patients & COSMIC \\
\hline R359L & In cancer patients & COSMIC, cBioPortal \\
\hline
\end{tabular}


Table 1. Cont

\begin{tabular}{|c|c|c|}
\hline Mutation & Mode of Identification & Reference \\
\hline E362K & In cancer patients & COSMIC, cBioPortal \\
\hline $\mathrm{E} 362^{*}$ & In cancer patients & COSMIC, TumorPortal \\
\hline F365C & In cancer patients & cBioPortal \\
\hline Q366H & In cancer patients & cBioPortal \\
\hline E367D & In cancer patients & COSMIC \\
\hline P373P & In cancer patients & COSMIC, TumorPortal \\
\hline $\mathrm{G}_{374}{ }^{*}$ & In cancer patients & COSMIC \\
\hline G374K & In cancer patients & COSMIC, cBioPortal \\
\hline L376R & In cancer patients & COSMIC, cBioPortal \\
\hline A378G & In cancer patients & COSMIC, cBioPortal \\
\hline \multicolumn{3}{|c|}{ (B) } \\
\hline $\mathrm{A} 2 \mathrm{~V}$ & In cancer patients & COSMIC, cBioPortal \\
\hline A6_A7delAA & In cancer patients & cosMIC \\
\hline A5delA & In cancer patients & COSMIC \\
\hline A7T & In cancer patients & COSMIC, cBioPortal \\
\hline G8S & In cancer patients & COSMIC, cBioPortal \\
\hline $\mathrm{R} 13 \mathrm{P}$ & In a screen for mutants that are resistant to VRT-11E and SCH772984 & [95] \\
\hline Q15Q & In cancer patients & COSMIC \\
\hline P21S & In cancer patients & COSMIC, cBioPortal \\
\hline $\mathrm{I} 29 \mathrm{M}$ & In Cancer patients, and in a screen for mutants that are resistant to VRT-11E & COSMIC, cBioPortal,[95] \\
\hline I29Q & In a screen for mutants that are resistant to VRT-11E & [95] \\
\hline I29R & In a screen for mutants that are resistant to VRT-11E & [95] \\
\hline I29L & In a screen for mutants that are resistant to VRT-11E & [95] \\
\hline $\mathrm{I} 29 \mathrm{E}$ & In a screen for mutants that are resistant to VRT-11E & [95] \\
\hline
\end{tabular}


Table 1. Cont.

\begin{tabular}{|c|c|c|}
\hline Mutation & Mode of Identification & Reference \\
\hline $\mathrm{I} 29 \mathrm{~K}$ & In a screen for mutants that are resistant to VRT-11E & [95] \\
\hline $\mathrm{I} 29 \mathrm{H}$ & In a screen for mutants that are resistant to VRT-11E & [95] \\
\hline I29Y & In a screen for mutants that are resistant to VRT-11E & [95] \\
\hline I29D & In a screen for mutants that are resistant to VRT-11E & [95] \\
\hline $\mathrm{I} 29 \mathrm{C}$ & In a screen for mutants that are resistant to VRT-11E & [95] \\
\hline $\mathrm{I} 29 \mathrm{~W}$ & In a screen for mutants that are resistant too VRT-11E & [95] \\
\hline $\mathrm{I} 29 \mathrm{~N}$ & In a screen for mutants that are resistant to VRT-11E & [95] \\
\hline G30P & In a screen for mutants that are resistant to VRT-11E & [95] \\
\hline E31P & In a screen for mutants that are resistant to SCH772984 & [95] \\
\hline E31Q & In cancer patients & COSMIC, cBioPortal \\
\hline G32D & In cancer patients & COSMIC, cBioPortal \\
\hline G32C & In cancer patients & cBioPortal \\
\hline $\mathrm{A} 33 \mathrm{~N}$ & In a screen for mutants that are resistant to VRT-11E & [95] \\
\hline Y34Y & In cancer patients & COSMIC \\
\hline $\mathrm{Y} 34 \mathrm{H}$ & In a screen for mutants that are resistant to VRT-11E and SCH772984 & {$[95,96]$} \\
\hline $\mathrm{Y} 34 \mathrm{~V}$ & In a screen for mutants that are resistant to VRT-11E and SCH772984 & [95] \\
\hline Y34T & In a screen for mutants that are resistant to VRT-11E and SCH772984 & [95] \\
\hline Y34Q & In a screen for mutants that are resistant to VRT-11E and SCH772984 & [95] \\
\hline Y34G & In a screen for mutants that are resistant to VRT-11E and SCH772984 & [95] \\
\hline Y34S & In a screen for mutants that are resistant to VRT-11E and SCH772984 & [95] \\
\hline $\mathrm{Y} 34 \mathrm{C}$ & In a screen for mutants that are resistant to VRT-11E and SCH772984 & [95] \\
\hline Y34I & In a screen for mutants that are resistant to VRT-11E and SCH772984 & [95] \\
\hline Y34D & In a screen for mutants that are resistant to VRT-11E and SCH772984 & [95] \\
\hline Y34R & In a screen for mutants that are resistant to VRT-11E and SCH772984 & [95] \\
\hline
\end{tabular}


Table 1. Cont

\begin{tabular}{|c|c|c|}
\hline Mutation & Mode of Identification & Reference \\
\hline $\mathrm{Y} 34 \mathrm{~N}$ & In a screen for mutants that are resistant to VRT-11E and SCH772984 & {$[95,96]$} \\
\hline Y34L & In a screen for mutants that are resistant to VRT-11E and SCH772984 & [95] \\
\hline Y34M & In a screen for mutants that are resistant to VRT-11E and SCH772984 & [95] \\
\hline G35D & In a screen for mutants that are resistant to VRT-11E and SCH772984 & [95] \\
\hline G35T & In a screen for mutants that are resistant to VRT-11E and SCH772984 & [95] \\
\hline G35K & In a screen for mutants that are resistant to VRT-11E and SCH772984 & [95] \\
\hline G35S & In a screen for mutants that are resistant to VRT-11E and SCH772984 & {$[95,96]$} \\
\hline G35A & In a screen for mutants that are resistant to VRT-11E and SCH772984 & [95] \\
\hline G35N & In a screen for mutants that are resistant to VRT-11E & [95] \\
\hline G35P & In a screen for mutants that are resistant to VRT-11E & [95] \\
\hline G35C & In a screen for mutants that are resistant to VRT-11E and SCH772984 & [95] \\
\hline M36P & In a screen for mutants that are resistant to VRT-11E & [95] \\
\hline V37A & In a screen for mutants that are resistant to VRT-11E & [95] \\
\hline $\mathrm{C} 38 \mathrm{P}$ & In a screen for mutants that are resistant to VRT-11E and SCH772984 & [95] \\
\hline C38R & In a screen for mutants that are resistant to SCH772984 & [95] \\
\hline Y41W & In a screen for mutants that are resistant to SCH772984 & [95] \\
\hline Y41E & In a screen for mutants that are resistant to SCH772984 & [95] \\
\hline $\mathrm{D} 42 \mathrm{H}$ & In a screen for mutants that are resistant to VRT-11E and SCH772984 & [95] \\
\hline V44F & In cancer patients & cBioPortal \\
\hline V47A & In a screen for mutants that are resistant to SCH772984 & [95] \\
\hline $\mathrm{R} 48^{*}$ & In cancer patients & COSMIC, cBioPortal \\
\hline $\mathrm{V} 49 \mathrm{~K}$ & Causes resistance to $\mathrm{SCH} 772984$ & [95] \\
\hline $\mathrm{V} 49 \mathrm{H}$ & In a screen for mutants that are resistant to VRT-11E and SCH772984 & [95] \\
\hline A50S & In Cancer patients & COSMIC, TumorPortal \\
\hline
\end{tabular}


Table 1. Cont

\begin{tabular}{|c|c|c|}
\hline Mutation & Mode of Identification & Reference \\
\hline K53G & In a screen for mutants that are resistant to SCH772984 & [95] \\
\hline $\mathrm{I} 54 \mathrm{H}$ & In a screen for mutants that are resistant to SCH772984 & [95] \\
\hline I54D & In a screen for mutants that are resistant to SCH772984 & [95] \\
\hline $54 \mathrm{~W}$ & In a screen for mutants that are resistant to SCH772984 & [95] \\
\hline I54K & In a screen for mutants that are resistant to SCH772984 & [95] \\
\hline I54Y & In a screen for mutants that are resistant to SCH772984 & [95] \\
\hline I54E & In a screen for mutants that are resistant to SCH772984 & [95] \\
\hline I54Q & In a screen for mutants that are resistant to VRT-11E and SCH772984 & [95] \\
\hline I54S & In a screen for mutants that are resistant to VRT-11E and SCH772984 & [95] \\
\hline I54G & In a screen for mutants that are resistant to VRT-11E and SCH772984 & [95] \\
\hline $\mathrm{I} 54 \mathrm{P}$ & In a screen for mutants that are resistant to VRT-11E & [95] \\
\hline S55P & In a screen for mutants that are resistant to VRT-11E & [95] \\
\hline S55G & In a screen for mutants that are resistant to VRT-11E & [95] \\
\hline S55F & In a screen for mutants that are resistant to VRT-11E and SCH772984 & [95] \\
\hline P56L & $\begin{array}{l}\text { In cancer patients, In a screen for mutants that are resistant to VRT-11E } \\
\text { and SCH772984 }\end{array}$ & COSMIC, cBioPortal, $[95,96]$ \\
\hline P56S & In a screen for mutants that are resistant to VRT-11E and SCH772984 & {$[95,96]$} \\
\hline P56W & In a screen for mutants that are resistant to VRT-11E and SCH772984 & [95] \\
\hline P56R & In a screen for mutants that are resistant to VRT-11E and SCH772984 & [95] \\
\hline P56K & In a screen for mutants that are resistant to VRT-11E and SCH772984 & [95] \\
\hline P56A & In a screen for mutants that are resistant to VRT-11E and SCH772984 & [95] \\
\hline P56M & In a screen for mutants that are resistant to VRT-11E and SCH772984 & [95] \\
\hline $\mathrm{P} 56 \mathrm{~N}$ & In a screen for mutants that are resistant to VRT-11E and SCH772984 & [95] \\
\hline
\end{tabular}


Table 1. Cont.

\begin{tabular}{|c|c|c|}
\hline Mutation & Mode of Identification & Reference \\
\hline P56G & In a screen for mutants that are resistant to VRT-11E and SCH772984 & [95] \\
\hline P56Y & In a screen for mutants that are resistant to VRT-11E and SCH772984 & [95] \\
\hline P56F & In a screen for mutants that are resistant to VRT-11E and SCH772984 & [95] \\
\hline P56Q & In a screen for mutants that are resistant to VRT-11E & [95] \\
\hline P56V & In a screen for mutants that are resistant to VRT-11E & [95] \\
\hline P56T & In a screen for mutants that are resistant to VRT-11E & {$[95,96]$} \\
\hline P56I & In a screen for mutants that are resistant to VRT-11E & [95] \\
\hline F57G & In a screen for mutants that are resistant to VRT-11E & [95] \\
\hline F57P & In a screen for mutants that are resistant to VRT-11E & [95] \\
\hline F57S & In a screen for mutants that are resistant to VRT-11E and SCH772984 & [95] \\
\hline F57R & In a screen for mutants that are resistant to VRT-11E and SCH772984 & [95] \\
\hline E58Q & In a screen for mutants that are resistant to SCH772984 & [95] \\
\hline E58S & In a screen for mutants that are resistant to SCH772984 & [95] \\
\hline Q60P & In a screen for mutants that are resistant to VRT-11E and SCH772984 & [95] \\
\hline $\mathrm{T} 61 \mathrm{~T}$ & In cancer patients & COSMIC \\
\hline T61I & In cancer patients & COSMIC \\
\hline Y62G & In a screen for mutants that are resistant to VRT-11E & [95] \\
\hline Y62E & In a screen for mutants that are resistant to VRT-11E & [95] \\
\hline Y62D & In a screen for mutants that are resistant to VRT-11E & [95] \\
\hline Y62S & In a screen for mutants that are resistant to VRT-11E & [95] \\
\hline $\mathrm{Y} 62 \mathrm{C}$ & In a screen for mutants that are resistant to VRT-11E & [95] \\
\hline $\mathrm{Y} 62 \mathrm{~T}$ & In a screen for mutants that are resistant to VRT-11E & [95] \\
\hline Y62Q & In a screen for mutants that are resistant to VRT-11E & [95] \\
\hline Y62A & In a screen for mutants that are resistant to VRT-11E & [95] \\
\hline
\end{tabular}


Table 1. Cont.

\begin{tabular}{|c|c|c|}
\hline Mutation & Mode of Identification & Reference \\
\hline Y62P & In a screen for mutants that are resistant to VRT-11E & [95] \\
\hline Y62V & In a screen for mutants that are resistant to VRT-11E and SCH772984 & [95] \\
\hline Y62M & In a screen for mutants that are resistant to VRT-11E and SCH772984 & [95] \\
\hline Y62K & In a screen for mutants that are resistant to VRT-11E and SCH772984 & [95] \\
\hline Y62I & In a screen for mutants that are resistance to VRT-11E and SCH772984 & [95] \\
\hline Y62L & In a screen for mutants that are resistance to VRT-11E and SCH772984 & [95] \\
\hline Y62R & In a screen for mutants that are resistant to VRT-11E and SCH772984 & [95] \\
\hline $\mathrm{Y} 62 \mathrm{~N}$ & In a screen for mutants that are resistant to VRT-11E & {$[95,96]$} \\
\hline $\mathrm{C} 63 \mathrm{~F}$ & In a screen for mutants that are resistant to VRT-11E & [95] \\
\hline C63W & In a screen for mutants that are resistant to VRT-11E & [95] \\
\hline $\mathrm{C} 63 \mathrm{fs} * 3$ & In Cancer patients & COSMIC, cBioPortal \\
\hline $\mathrm{C} 63 \mathrm{Y}$ & In a screen for mutants that are resistant to VRT-11E and SCH772984 & {$[95,96]$} \\
\hline R65I & In cancer patients, and in a screen for mutants that are resistant to VRT-11E & COSMIC, cBioPortal,[95] \\
\hline $\mathrm{R} 65 \mathrm{~K}$ & $\begin{array}{l}\text { In a screen for mutants that are resistant to Erk inhibitors and } \\
\text { RAF/MEK inhibitors }\end{array}$ & [96] \\
\hline R65S & Genetic screen for Mpk1 intrinsically active mutants & [73] \\
\hline $\mathrm{T} 66 \mathrm{~T}$ & In Cancer patients & COSMIC \\
\hline $\mathrm{T} 66 \mathrm{M}$ & In a screen for mutants that are resistant to VRT-11E & {$[95,96]$} \\
\hline T66Q & In a screen for mutants that are resistant to VRT-11E & {$[95,96]$} \\
\hline $\mathrm{T} 66 \mathrm{~F}$ & In a screen for mutants that are resistant to VRT-11E & {$[95,96]$} \\
\hline T66I & In a screen for mutants that are resistant to VRT-11E & {$[95,96]$} \\
\hline $\mathrm{T} 66 \mathrm{~L}$ & In a screen for mutants that are resistant to VRT-11E & {$[95,96]$} \\
\hline $\mathrm{T} 66 \mathrm{P}$ & In a screen for mutants that are resistant to VRT-11E & {$[95,96]$} \\
\hline
\end{tabular}


Table 1. Cont.

\begin{tabular}{|c|c|c|}
\hline Mutation & Mode of Identification & Reference \\
\hline T66D & In a screen for mutants that are resistant to VRT-11E and SCH772984 & {$[95,96]$} \\
\hline T66Y & In a screen for mutants that are resistant to VRT-11E and SCH772984 & {$[95,96]$} \\
\hline $\mathrm{T} 66 \mathrm{H}$ & In a screen for mutants that are resistant to VRT-11E & {$[95,96]$} \\
\hline $\mathrm{T} 66 \mathrm{~N}$ & In a screen for mutants that are resistant to VRT-11E and SCH772984 & {$[95,96]$} \\
\hline L67L & In cancer patients & COSMIC \\
\hline R68R & In cancer patients & COSMIC \\
\hline E69P & In a screen for mutants that are resistant to VRT-11E & {$[95,96]$} \\
\hline E69C & In a screen for mutants that are resistant to VRT-11E & {$[95,96]$} \\
\hline E69G & In a screen for mutants that are resistant to VRT-11E & {$[95,96]$} \\
\hline $\mathrm{E} 69 \mathrm{~K}$ & In a screen for mutants that are resistant to VRT-11E and SCH772984 & {$[95,96]$} \\
\hline E69A & In a screen for mutants that are resistant to VRT-11E and SCH772984 & {$[95,96]$} \\
\hline $\mathrm{I} 72 \mathrm{fs} * 8$ & In cancer patients & COSMIC \\
\hline $\mathrm{L} 73 \mathrm{E}$ & In a screen for mutants that are resistant to VRT-11E & {$[95,96]$} \\
\hline $\mathrm{L} 73 \mathrm{H}$ & In a screen for mutants that are resistant to VRT-11E & {$[95,96]$} \\
\hline L73R & In a screen for mutants that are resistant to VRT-11E & {$[95,96]$} \\
\hline L73W & In a screen for mutants that are resistant to VRT-11E and SCH772984 & [95] \\
\hline L73P & $\begin{array}{l}\text { In a screen for mutants that are resistant to VRT-11E, and on the basis of } \\
\text { genetic screen for intrinsically active FUS3 }\end{array}$ & {$[95,103]$} \\
\hline $\mathrm{R} 75 \mathrm{C}$ & In cancer patients & COSMIC, cBioPortal \\
\hline R77S & In cancer patients & cBioPortal \\
\hline R77K & In cancer patients & COSMIC, cBioPortal \\
\hline E79K & In cancer patients & COSMIC, cBioPortal, TumorPortal \\
\hline $\mathrm{N} 80 \mathrm{fs} * 18$ & In cancer patients & COSMIC, cBioPortal \\
\hline $\mathrm{I} 82 \mathrm{~T}$ & In cancer patients & COSMIC, cBioPortal \\
\hline I82A & Activating mutation. On the basis of structural considerations & [76] \\
\hline
\end{tabular}


Table 1. Cont.

\begin{tabular}{|c|c|c|}
\hline Mutation & Mode of Identification & Reference \\
\hline I84A & Activating mutation. On the basis of structural considerations & [76] \\
\hline D86-del & In cancer patients & cBioPortal \\
\hline $\mathrm{I} 88 \mathrm{~F}$ & In cancer patients & COSMIC \\
\hline I93I & In cancer patients & COSMIC, TumorPortal \\
\hline Q95R & In a screen for mutants that are resistant to VRT-11E & [95] \\
\hline M96W & In a screen for mutants that are resistant to VRT-11E & [95] \\
\hline M96I & In cancer patients & cBioPortal \\
\hline $\mathrm{D} 98 \mathrm{~N}$ & In cancer patients & cBioPortal \\
\hline $\mathrm{D} 98 \mathrm{M}$ & In a screen for mutants that are resistant to VRT-11E & [95] \\
\hline I101Q & In a screen for mutants that are resistant to SCH772984 & [95] \\
\hline I101W & In a screen for mutants that are resistant to SCH772984 & [95] \\
\hline I101Y & In a screen for mutants that are resistant to SCH772984 & [95] \\
\hline I101R & In cancer patients & COSMIC \\
\hline V102V & $\begin{array}{l}\text { In a screen for mutants that are resistant to Erk inhibitors and } \\
\text { RAF/MEK inhibitors }\end{array}$ & [96] \\
\hline Q103A & Generated in order to study the biological effect the gatekeeper residue & [76] \\
\hline Q103G & Generated in order to study the biological effect the gatekeeper residue & [76] \\
\hline Q103I & In a screen for mutants that are resistant to SCH772984 & [95] \\
\hline Q103F & In a screen for mutants that are resistant to SCH772984 & [95] \\
\hline Q103T & In a screen for mutants that are resistant to SCH772984 & [95] \\
\hline Q103W & In a screen for mutants that are resistant to VRT-11E and SCH772984 & [95] \\
\hline Q103V & In a screen for mutants that are resistant to VRT-11E and SCH772984 & [95] \\
\hline Q103N & In a screen for mutants that are resistant to VRT-11E & [95] \\
\hline Q103Y & In a screen for mutants that are resistant to VRT-11E and SCH772984 & [95] \\
\hline D104D & In a screen for mutants that are resistant to Erk inhibitors & [96] \\
\hline
\end{tabular}


Table 1. Cont

\begin{tabular}{|c|c|c|}
\hline Mutation & Mode of Identification & Reference \\
\hline $\mathrm{D} 104 \mathrm{H}$ & In cancer patients & cBioPortal \\
\hline $\mathrm{T} 108 \mathrm{P}$ & In a screen for mutants that are resistant to VRT-11E & [95] \\
\hline L110R & In cancer patients & cBioPortal \\
\hline L114S & In cancer patients & cBioPortal \\
\hline L119I & In cancer patients & COSMIC, cBioPortal, TumorPortal \\
\hline $\mathrm{D} 122 \mathrm{~T}$ & In a screen for mutants that are resistant to VRT-11E and SCH772984 & [95] \\
\hline $\mathrm{I} 124 \mathrm{~F}$ & In cancer patients & COSMIC \\
\hline C125I & In a screen for mutants that are resistant to VRT-11E and SCH772984 & [95] \\
\hline $\mathrm{Y} 129 \mathrm{~N}$ & In a screen for mutants that are resistant to RAF/MEK inhibitors & [96] \\
\hline $\mathrm{Y} 129 \mathrm{H}$ & In a screen for mutants that are resistant to RAF/MEK inhibitors & [96] \\
\hline Y129F & In a screen for mutants that are resistant to trametinib and dabrafenib & [96] \\
\hline Y129C & $\begin{array}{l}\text { In Cancer patients, and In a screen for mutants that are resistant to trametinib } \\
\text { and dabrafenib }\end{array}$ & COSMIC, cBioPortal, [96] \\
\hline Y129S & In a screen for mutants that are resistant totrametinib and dabrafenib & [96] \\
\hline Q130E & In cancer patients & COSMIC, cBioPortal \\
\hline L132P & In cancer patients & COSMIC, cBioPortal \\
\hline R133K & In cancer patients & COSMIC, cBioPortal \\
\hline G134E & In cancer patients & COSMIC, cBioPortal \\
\hline I138I & In cancer patients & COSMIC \\
\hline H139Y & In cancer patients & COSMIC, cBioPortal, TumorPortal \\
\hline H139R & In cancer patients & cBioPortal \\
\hline S140L & In cancer patients & COSMIC, cBioPortal \\
\hline A141A & In cancer patients & COSMIC, TumorPortal \\
\hline N142K & In cancer patients & COSMIC, cBioPortal \\
\hline $\mathrm{N} 142 \mathrm{~N}$ & In cancer patients & COSMIC \\
\hline
\end{tabular}


Table 1. Cont

\begin{tabular}{|c|c|c|}
\hline Mutation & Mode of Identification & Reference \\
\hline H145Y & In cancer patients & COSMIC, cBioPortal \\
\hline H145R & In cancer patients & cBioPortal \\
\hline R146S & In cancer patients & COSMIC, cBioPortal \\
\hline R146L & In cancer patients & cBioPortal, TumorPortal \\
\hline R146C & In cancer patients & cBioPortal \\
\hline $\mathrm{R} 146 \mathrm{H}$ & In cancer patients & COSMIC, cBioPortal \\
\hline D147Y & In cancer patients & cBioPortal \\
\hline S151D & On the basis of alignment with MKK1 & [103] \\
\hline L154N & In a screen for mutants that are resistant to VRT-11E & [95] \\
\hline L154G & In a screen for mutants that are resistant to VRT-11E & [95] \\
\hline L155L & In cancer patients & COSMIC, TumorPortal \\
\hline $\mathrm{D} 160 \mathrm{~N}$ & In cancer patients & COSMIC, cBioPortal, TumorPortal \\
\hline D160G & In cancer patients & COSMIC, cBioPortal, TumorPortal \\
\hline I163I & In cancer patients & cBioPortal \\
\hline C164R & In cancer patients & COSMIC, cBioPortal \\
\hline D165G & In cancer patients & COSMIC, cBioPortal \\
\hline G167D & In a screen for mutants that are resistant to VRT-11E and SCH772984 & {$[95,97]$} \\
\hline L168L & In a screen for mutants that are resistant to Erk inhibitors & [96] \\
\hline $\mathrm{R} 170 \mathrm{H}$ & In cancer patients & COSMIC, cBioPortal, TumorPortal \\
\hline V171I & In cancer patients & COSMIC \\
\hline P174T & In cancer patients & COSMIC, cBioPortal \\
\hline P174S & In cancer patients & COSMIC, cBioPortal \\
\hline D177G & In cancer patients & cBioPortal \\
\hline $\mathrm{T} 179 \mathrm{fs}^{*} 29$ & In cancer patients & COSMIC, cBioPortal \\
\hline F181S & In cancer patients & COSMIC \\
\hline
\end{tabular}


Table 1. Cont

\begin{tabular}{|c|c|c|}
\hline Mutation & Mode of Identification & Reference \\
\hline E184* & In cancer patients & cBioPortal \\
\hline A187V & In a screen for mutants that are resistant to trametinib and dabrafenib & [96] \\
\hline $\mathrm{R} 189 \mathrm{C}$ & In cancer patients & COSMIC, cBioPortal \\
\hline $\mathrm{R} 189 \mathrm{H}$ & In cancer patients & COSMIC, cBioPortal \\
\hline W190L & In cancer patients & cBioPortal \\
\hline E195* & In cancer patients & cBioPortal \\
\hline L198F & In cancer patients & COSMIC \\
\hline S200P & In a screen for mutants that are resistant to trametinib and dabrafenib & [96] \\
\hline G202C & In cancer patients & COSMIC, cBioPortal,[96] \\
\hline G202S & In cancer patients & cBioPortal,[96] \\
\hline G202G & In cancer patients & COSMIC, [96] \\
\hline Y203N & In a screen for mutants that are resistant to RAF/MEK inhibitors & [96] \\
\hline T204I & In cancer patients & COSMIC, cBioPortal \\
\hline $\mathrm{I} 209 \mathrm{~V}$ & In cancer patients & COSMIC, cBioPortal \\
\hline $\mathrm{V} 212 \mathrm{~A}$ & In a screen for mutants that are resistant to RAF/MEK inhibitors & [96] \\
\hline E218K & In cancer patients & COSMIC \\
\hline S220Y & In cancer patients & COSMIC, cBioPortal \\
\hline $\mathrm{I} 225 \mathrm{~F}$ & In cancer patients & COSMIC, cBioPortal \\
\hline P227S & In cancer patients & COSMIC \\
\hline $\mathrm{P} 227 \mathrm{~L}$ & In cancer patients & COSMIC, cBioPortal \\
\hline G228E & In cancer patients & COSMIC, cBioPortal \\
\hline D233E & In cancer patients & COSMIC, cBioPortal \\
\hline D233G & In cancer patients & cBioPortal \\
\hline
\end{tabular}


Table 1. Cont.

\begin{tabular}{|c|c|c|}
\hline Mutation & Mode of Identification & Reference \\
\hline D233V & In cancer patients & COSMIC, cBioPortal \\
\hline D233* & In cancer patients & TumorPortal \\
\hline G240_splice & In cancer patients & cBioPortal \\
\hline L242I & In cancer patients & COSMIC, cBioPortal \\
\hline L242F & In cancer patients & COSMIC, cBioPortal, TumorPortal \\
\hline S244F & In cancer patients & COSMIC, cBioPortal \\
\hline S244S & In cancer patients & COSMIC \\
\hline L250L & In a screen for mutants that are resistant to RAF/MEK inhibitors & [96] \\
\hline N255S & In cancer patients & COSMIC, cBioPortal \\
\hline R259G & In cancer patients & COSMIC, cBioPortal \\
\hline N260T & In cancer patients & COSMIC, cBioPortal, TumorPortal \\
\hline Y261C & In cancer patients & COSMIC, cBioPortal \\
\hline L263F & In a screen for mutants that are resistant to Erk inhibitors & [96] \\
\hline S264F & In cancer patients & COSMIC \\
\hline L265P & In cancer patients & COSMIC \\
\hline P266L & In cancer patients & COSMIC, cBioPortal \\
\hline $\mathrm{P} 272 \mathrm{~S}$ & In cancer patients & COSMIC \\
\hline L276L & In cancer patients & COSMIC, TumorPortal \\
\hline L276M & In cancer patients & cBioPortal \\
\hline $\mathrm{F} 277 \mathrm{~F}$ & In cancer patients & COSMIC \\
\hline K283T & In cancer patients & cBioPortal \\
\hline L285M & In a screen for mutants that are resistant to RAF/MEK inhibitors & [96] \\
\hline L288L & In cancer patients & COSMIC \\
\hline D289G & In cancer patients & COSMIC, cBioPortal, TumorPortal \\
\hline $\mathrm{D} 289 \mathrm{H}$ & In cancer patients & cBioPortal \\
\hline
\end{tabular}


Table 1. Cont

\begin{tabular}{|c|c|c|}
\hline Mutation & Mode of Identification & Reference \\
\hline P296T & In cancer patients & COSMIC \\
\hline E301K & In a screen for mutants that are resistant to RAF/MEK inhibitors & [96] \\
\hline A305S & In cancer patients & cBioPortal \\
\hline L311P & In cancer patients & COSMIC \\
\hline Y314F & In cancer patients & COSMIC, cBioPortal, TumorPortal \\
\hline Y314C & In cancer patients & cBioPortal \\
\hline D316N & In cancer patients & COSMIC, cBioPortal \\
\hline P317S & In cancer patients & COSMIC, cBioPortal \\
\hline P317P & In cancer patients & COSMIC \\
\hline S318C & In cancer patients & COSMIC \\
\hline D319N & In cancer patients, and in a genetic screen in Drosophila & COSMIC, cBioPortal, [77] \\
\hline D319A & In cancer patients & COSMIC \\
\hline D319G & In a screen for mutants that are resistant to trametinib and dabrafenib & cBioPortal, [96] \\
\hline D319V & In cancer patients & COSMIC, cBioPortal \\
\hline D319E & In cancer patients & COSMIC, cBioPortal \\
\hline E320K & In a screen for mutants that are resistant to trametinib and dabrafenib & CISMIC, cBioPortal, TumorPortal,[96] \\
\hline $\mathrm{E} 320^{*}$ & In cancer patients & cBioPortal, TumorPortal \\
\hline $\mathrm{E} 320 \mathrm{~N}$ & In cancer patients & cBioPortal \\
\hline E320A & In cancer patients & COSMIC, cBioPortal \\
\hline E320V & In cancer patients & COSMIC \\
\hline P321P & In a screen for mutants that are resistant to Erk inhibitors & [96] \\
\hline P321S & In a screen for mutants that are resistant to RAF/MEK inhibitors & [96] \\
\hline P321L & In a screen for mutants that are resistant to RAF/MEK inhibitors & [96] \\
\hline $\mathrm{I} 322 \mathrm{~V}$ & In cancer patients & cBioPortal \\
\hline
\end{tabular}


Table 1. Cont.

\begin{tabular}{|c|c|c|}
\hline Mutation & Mode of Identification & Reference \\
\hline $\mathrm{A} 323 \mathrm{~T}$ & In cancer patients & cBioPortal \\
\hline A323S & In cancer patients & cBioPortal \\
\hline $\mathrm{A} 323 \mathrm{~V}$ & In cancer patients & cBioPortal \\
\hline $\mathrm{E} 324^{*}$ & In cancer patients & COSMIC, cBioPortal \\
\hline F329F & In cancer patients & COSMIC \\
\hline F329Y & In cancer patients & COSMIC \\
\hline D330N & In cancer patients & COSMIC, cBioPortal \\
\hline M331I & In cancer patients & COSMIC, cBioPortal \\
\hline D335N & In a screen for mutants that are resistant to RAF/MEK inhibitors & [96] \\
\hline E343* & In cancer patients & COSMIC \\
\hline $\mathrm{I} 345 \mathrm{H}$ & In a screen for mutants that are resistant to to VRT-11E & [95] \\
\hline $\mathrm{I} 345 \mathrm{M}$ & In a screen for mutants that are resistant to VRT-11E & [95] \\
\hline $\mathrm{I} 345 \mathrm{~F}$ & In a screen for mutants that are resistant to VRT-11E & [95] \\
\hline I345L & In a screen for mutants that are resistant to VRT-11E & COSMIC \\
\hline I345Y & In a screen for mutants that are resistant to VRT-11E and SCH772984 & [95] \\
\hline I345W & In a screen for mutants that are resistant to VRT-11E and SCH772984 & [95] \\
\hline $\mathrm{E} 347^{*}$ & In cancer patients & COSMIC \\
\hline E347K & $\begin{array}{l}\text { In cancer patients, and in a screen for mutants that are resistant to } \\
\text { RAF/MEK inhibitors }\end{array}$ & COSMIC, cBioPortal,[96] \\
\hline T349T & In cancer patients & COSMIC \\
\hline A350S & In cancer patients & COSMIC \\
\hline $\mathrm{A} 350 \mathrm{~V}$ & In cancer patients & COSMIC \\
\hline $\mathrm{R} 351 \mathrm{~K}$ & In a screen for mutants that are resistant to RAF/MEK inhibitors & [96] \\
\hline Y356D & In cancer patients & COSMIC, cBioPortal \\
\hline Y356Y & In cancer patients & COSMIC \\
\hline $\mathrm{R} 357 \mathrm{~T}$ & In cancer patients & cBioPortal \\
\hline
\end{tabular}


Funding: Research in David Engelberg laboratory was funded by the Israel Science Foundation grant number $(1463 / 18)$ and by the Singapore National Research Foundation under its HUJ-NUS partnership program at the Campus for Research Excellence and Technology Enterprise (CREATE). David Engelberg holds a Wolfson Family Chair in Biochemistry.

Conflicts of Interest: The authors declare no conflict of interest.

\section{References}

1. Askari, N.; Diskin, R.; Avitzour, M.; Capone, R.; Livnah, O.; Engelberg, D. Hyperactive variants of p38 $\alpha$ induce, whereas hyperactive variants of $\mathrm{p} 38 \gamma$ suppress, activating protein 1-mediated transcription. J. Biol. Chem. 2007, 282, 91-99. [CrossRef] [PubMed]

2. Avitzour, M.; Diskin, R.; Raboy, B.; Askari, N.; Engelberg, D.; Livnah, O. Intrinsically active variants of all human p38 isoforms. FEBS J. 2007, 274, 963-975. [CrossRef] [PubMed]

3. Fujimura, T. Current status and future perspective of robot-assisted radical cystectomy for invasive bladder cancer. Int. J. Urol. 2019, 26, 1033-1042. [CrossRef] [PubMed]

4. Roskoski, R., Jr. Targeting oncogenic Raf protein-serine/threonine kinases in human cancers. Pharmacol. Res. 2018, 135, 239-258. [CrossRef]

5. Keith, W.M.; Kenichi, N.; Sara, W. Recent advances of MEK inhibitors and their clinical progress. Curr. Top. Med. Chem. 2007, 7, 1364-1378.

6. Zhao, Y.; Adjei, A.A. The clinical development of MEK inhibitors. Nat. Rev. Clin. Oncol. 2014, 11, 385-400. [CrossRef]

7. Roskoski, R., Jr. Allosteric MEK1/2 inhibitors including cobimetanib and trametinib in the treatment of cutaneous melanomas. Pharmacol. Res. 2017, 117, 20-31. [CrossRef]

8. Dominguez, C.; Powers, D.A.; Tamayo, N. p38 MAP kinase inhibitors: Many are made, but few are chosen. Curr. Opin. Drug Discov. Dev. 2005, 8, 421-430.

9. Yong, H.Y.; Koh, M.S.; Moon, A. The p38 MAPK inhibitors for the treatment of inflammatory diseases and cancer. Expert Opin. Investig. Drugs 2009, 18, 1893-1905. [CrossRef]

10. Pettus, L.H.; Wurz, R.P. Small molecule p 38 MAP kinase inhibitors for the treatment of inflammatory diseases: Novel structures and developments during 2006-2008. Curr. Top. Med. Chem. 2008, 8, 1452-1467. [CrossRef]

11. Messoussi, A.; Feneyrolles, C.; Bros, A.; Deroide, A.; Daydé-Cazals, B.; Chevé, G.; Van Hijfte, N.; Fauvel, B.; Bougrin, K.; Yasri, A. Recent progress in the design, study, and development of c-Jun N-terminal kinase inhibitors as anticancer agents. Chem. Biol. 2014, 21, 1433-1443. [CrossRef] [PubMed]

12. Mansour, S.J.; Matten, W.T.; Hermann, A.S.; Candia, J.M.; Rong, S.; Fukasawa, K.; Woude, G.V.; Ahn, N.G. Transformation of mammalian cells by constitutively active MAP kinase kinase. Science 1994, 265, 966-970. [CrossRef] [PubMed]

13. Kolibaba, K.S.; Druker, B.J. Protein tyrosine kinases and cancer. Biochim. Biophys. Acta 1997, 1333, F217-F248. [CrossRef]

14. Garnett, M.J.; Marais, R. Guilty as charged: B-RAF is a human oncogene. Cancer Cell 2004, 6, 313-319. [CrossRef] [PubMed]

15. Raingeaud, J.; Whitmarsh, A.J.; Barrett, T.; Derijard, B.; Davis, R.J. MKK3-and MKK6-regulated gene expression is mediated by the $\mathrm{p} 38$ mitogen-activated protein kinase signal transduction pathway. Mol. Cell. Biol. 1996, 16, 1247-1255. [CrossRef] [PubMed]

16. Wick, M.J.; Wick, K.R.; Chen, H.; He, H.; Dong, L.Q.; Quon, M.J.; Liu, F. Substitution of the autophosphorylation site Thr516 with a negatively charged residue confers constitutive activity to mouse 3-phosphoinositide-dependent protein kinase-1 in cells. J. Biol. Chem. 2002, 277, 16632-16638. [CrossRef]

17. Yang, L.; Zheng, L.; Chng, W.J.; Ding, J.L. Comprehensive Analysis of ERK1/2 Substrates for Potential Combination Immunotherapies. Trends Pharmacol. Sci. 2019, 40, 897-910. [CrossRef]

18. Kyriakis, J.M.; Avruch, J. Mammalian MAPK signal transduction pathways activated by stress and inflammation: A 10-year update. Physiol. Rev. 2012, 92, 689-737. [CrossRef]

19. Brewster, J.L.; Gustin, M.C. Hog1: 20 years of discovery and impact. Sci. Signal 2014, 7, re7. [CrossRef]

20. Engelberg, D.; Perlman, R.; Levitzki, A. Transmembrane signaling in Saccharomyces cerevisiae as a model for signaling in metazoans: State of the art after 25 years. Cell. Signal. 2014, 26, 2865-2878. [CrossRef] 
21. Saito, H. Regulation of cross-talk in yeast MAPK signaling pathways. Curr. Opin. Microbiol. 2010, 13, 677-683. [CrossRef] [PubMed]

22. Chen, R.E.; Thorner, J. Function and regulation in MAPK signaling pathways: Lessons learned from the yeast Saccharomyces cerevisiae. Biochim. Biophys. Acta 2007, 1773, 1311-1340. [CrossRef] [PubMed]

23. Shilo, B.Z. The regulation and functions of MAPK pathways in Drosophila. Methods 2014, 68, 151-159. [CrossRef] [PubMed]

24. Ashton-Beaucage, D.; Therrien, M. How Genetics Has Helped Piece Together the MAPK Signaling Pathway; Humana Press: New York, NY, USA, 2017; Volume 1487, pp. 1-21.

25. Bost, F.; Aouadi, M.; Caron, L.; Even, P.; Belmonte, N.; Prot, M.; Dani, C.; Hofman, P.; Pagès, G.; Pouysségur, J.; et al. The extracellular signal-regulated kinase isoform ERK1 is specifically required for in vitro and in vivo adipogenesis. Diabetes 2005, 54, 402-411. [CrossRef] [PubMed]

26. Bourcier, C.; Jacquel, A.; Hess, J.; Peyrottes, I.; Angel, P.; Hofman, P.; Auberger, P.; Pouysségur, J.; Pagès, G. p44 mitogen-activated protein kinase (extracellular signal-regulated kinase 1)-dependent signaling contributes to epithelial skin carcinogenesis. Cancer Res. 2006, 66, 2700-2707. [CrossRef] [PubMed]

27. Guihard, S.; Clay, D.; Cocault, L.; Saulnier, N.; Opolon, P.; Souyri, M.; Pagès, G.; Pouysségur, J.; Porteu, F.; Gaudry, M. The MAPK ERK1 is a negative regulator of the adult steady-state splenic erythropoiesis. Blood 2010, 115, 3686-3694. [CrossRef]

28. Lefloch, R.; Pouysségur, J.; Lenormand, P. Single and combined silencing of ERK1 and ERK2 reveals their positive contribution to growth signaling depending on their expression levels. Mol. Cell. Biol. 2008, 28, 511-527. [CrossRef]

29. Shin, S.; Dimitri, C.A.; Yoon, S.O.; Dowdle, W.; Blenis, J. ERK2 but not ERK1 induces epithelial-to-mesenchymal transformation via DEF motif-dependent signaling events. Mol. Cell 2010, 38, 114-127. [CrossRef]

30. Voisin, L.; Saba-El-Leil, M.K.; Julien, C.; Frémin, C.; Meloche, S. Genetic demonstration of a redundant role of extracellular signal-regulated kinase 1 (ERK1) and ERK2 mitogen-activated protein kinases in promoting fibroblast proliferation. Mol. Cell. Biol. 2010, 30, 2918-2932. [CrossRef]

31. Frémin, C.; Ezan, F.; Boisselier, P.; Bessard, A.; Pagès, G.; Pouysségur, J.; Baffet, G. ERK2 but not ERK1 plays a key role in hepatocyte replication: An RNAi-mediated ERK2 knockdown approach in wild-type and ERK1 null hepatocytes. Hepatology 2007, 45, 1035-1045. [CrossRef]

32. Radtke, S.; Milanovic, M.; Rossé, C.; De Rycker, M.; Lachmann, S.; Hibbert, A.; Kermorgant, S.; Parker, P.J. ERK2 but not ERK1 mediates HGF-induced motility in non-small cell lung carcinoma cell lines. J. Cell Sci. 2013, 126 Pt 11, 2381-2391. [CrossRef]

33. Chang, S.F.; Lin, S.S.; Yang, H.C.; Chou, Y.Y.; Gao, J.I.; Lu, S.C. LPS-Induced G-CSF Expression in Macrophages Is Mediated by ERK2, but Not ERK1. PLoS ONE 2015, 10, e0129685. [CrossRef] [PubMed]

34. Samuels, I.S.; Karlo, J.C.; Faruzzi, A.N.; Pickering, K.; Herrup, K.; Sweatt, J.D.; Saitta, S.C.; Landreth, G.E. Deletion of ERK2 mitogen-activated protein kinase identifies its key roles in cortical neurogenesis and cognitive function. J. Neurosci. 2008, 28, 6983-6995. [CrossRef] [PubMed]

35. Hatano, N.; Mori, Y.; Oh-hora, M.; Kosugi, A.; Fujikawa, T.; Nakai, N.; Niwa, H.; Miyazaki, J.I.; Hamaoka, T.; Ogata, M. Essential role for ERK2 mitogen-activated protein kinase in placental development. Genes Cells 2003, 8, 847-856. [CrossRef] [PubMed]

36. Frémin, C.; Saba-El-Leil, M.K.; Lévesque, K.; Ang, S.L.; Meloche, S. Functional Redundancy of ERK1 and ERK2 MAP Kinases during Development. Cell Rep. 2015, 12, 913-921. [CrossRef]

37. Marshall, C.J. Specificity of receptor tyrosine kinase signaling: Transient versus sustained extracellular signal-regulated kinase activation. Cell 1995, 80, 179-185. [CrossRef]

38. McKay, M.M.; Morrison, D.K. Integrating signals from RTKs to ERK/MAPK. Oncogene 2007, 26, 3113-3121. [CrossRef]

39. Wee, P.; Wang, Z. Epidermal Growth Factor Receptor Cell Proliferation Signaling Pathways. Cancers 2017, 9, 52.

40. Katz, M.; Amit, I.; Yarden, Y. Regulation of MAPKs by growth factors and receptor tyrosine kinases. Biochim. Biophys. Acta 2007, 1773, 1161-1176. [CrossRef]

41. Schlessinger, J. Receptor tyrosine kinases: Legacy of the first two decades. Cold Spring Harb. Perspect. Biol. 2014, 6, a008912. [CrossRef]

42. Liu, F.; Yang, X.; Geng, M.; Huang, M. Targeting ERK, an Achilles' Heel of the MAPK pathway, in cancer therapy. Acta Pharm. Sin. B 2018, 8, 552-562. [CrossRef] [PubMed] 
43. Lemmon, M.A.; Schlessinger, J. Cell signaling by receptor tyrosine kinases. Cell 2010, 141, 1117-1134. [CrossRef] [PubMed]

44. van der Geer, P.; Hunter, T.; Lindberg, R.A. Receptor Protein-Tyrosine Kinases and Their Signal-Transduction Pathways. Annu. Rev. Cell Biol. 1994, 10, 251-337. [CrossRef] [PubMed]

45. Wellbrock, C.; Karasarides, M.; Marais, R. The RAF proteins take centre stage. Nat. Rev. Mol. Cell Biol. 2004, 5, 875-885. [CrossRef] [PubMed]

46. Okazaki, K.; Sagata, N. The Mos/MAP kinase pathway stabilizes c-Fos by phosphorylation and augments its transforming activity in NIH 3 T3 cells. EMBO J. 1995, 14, 5048-5059. [CrossRef]

47. Das, S.; Cho, J.; Lambertz, I.; Kelliher, M.A.; Eliopoulos, A.G.; Du, K.; Tsichlis, P.N. Tpl2/cot signals activate ERK, JNK, and NF-kappaB in a cell-type and stimulus-specific manner. J. Biol. Chem. 2005, 280, 23748-23757. [CrossRef]

48. Gotoh, I.; Adachi, M.; Nishida, E. Identification and characterization of a novel MAP kinase kinase kinase, MLTK. J. Biol. Chem. 2001, 276, 4276-4286. [CrossRef]

49. Shenoy, S.K.; Drake, M.T.; Nelson, C.D.; Houtz, D.A.; Xiao, K.; Madabushi, S.; Reiter, E.; Premont, R.T.; Lichtarge, O.; Lefkowitz, R.J. beta-arrestin-dependent, $\mathrm{G}$ protein-independent ERK1/2 activation by the beta2 adrenergic receptor. J. Biol. Chem. 2006, 281, 1261-1273. [CrossRef]

50. Choi, M.; Staus, D.P.; Wingler, L.M.; Ahn, S.; Pani, B.; Capel, W.D.; Lefkowitz, R.J. G protein-coupled receptor kinases (GRKs) orchestrate biased agonism at the beta2-adrenergic receptor. Sci. Signal 2018, 11, eaar7084. [CrossRef]

51. Jain, R.; Watson, U.; Vasudevan, L.; Saini, D.K. ERK Activation Pathways Downstream of GPCRs. Int. Rev. Cell Mol. Biol. 2018, 338, 79-109.

52. Watson, U.; Jain, R.; Asthana, S.; Saini, D.K. Spatiotemporal Modulation of ERK Activation by GPCRs. Int. Rev. Cell Mol. Biol. 2018, 338, 111-140. [PubMed]

53. Morrison, D.K. KSR: A MAPK scaffold of the Ras pathway? J. Cell Sci. 2001, 114, 1609-1612. [PubMed]

54. Sharma, C.; Vomastek, T.; Tarcsafalvi, A.; Catling, A.D.; Schaeffer, H.J.; Eblen, S.T.; Weber, M.J. MEK partner 1 (MP1): Regulation of oligomerization in MAP kinase signaling. J. Cell. Biochem. 2005, 94, 708-719. [CrossRef] [PubMed]

55. Morrison, D.K.; Davis, R.J. Regulation of MAP kinase signaling modules by scaffold proteins in mammals. Annu. Rev. Cell Dev. Biol. 2003, 19, 91-118. [CrossRef] [PubMed]

56. Frodin, M.; Gammeltoft, S. Role and regulation of 90 kDa ribosomal S6 kinase (RSK) in signal transduction. Mol. Cell. Endocrinol. 1999, 151, 65-77. [CrossRef]

57. Reyskens, K.M.; Arthur, J.S.C. Emerging Roles of the Mitogen and Stress Activated Kinases MSK1 and MSK2. Front. Cell Dev. Biol. 2016, 4, 56. [CrossRef]

58. Gaestel, M. Specificity of signaling from MAPKs to MAPKAPKs: kinases' tango nuevo. Front. Biosci. 2008, 13, 6050-6059. [CrossRef]

59. Cruzalegui, F.H.; Cano, E.; Treisman, R. ERK activation induces phosphorylation of Elk-1 at multiple S/T-P motifs to high stoichiometry. Oncogene 1999, 18, 7948-7957. [CrossRef]

60. Dougherty, M.K.; Müller, J.; Ritt, D.A.; Zhou, M.; Zhou, X.Z.; Copeland, T.D.; Conrads, T.P.; Veenstra, T.D.; Lu, K.P.; Morrison, D.K. Regulation of Raf-1 by direct feedback phosphorylation. Mol. Cell 2005, 17, $215-224$. [CrossRef]

61. Fritsche-Guenther, R.; Witzel, F.; Sieber, A.; Herr, R.; Schmidt, N.; Braun, S.; Brummer, T.; Sers, C.; Blüthgen, N. Strong negative feedback from Erk to Raf confers robustness to MAPK signalling. Mol. Syst. Biol. 2011, 7, 489. [CrossRef]

62. Shin, S.Y.; Rath, O.; Choo, S.M.; Fee, F.; McFerran, B.; Kolch, W.; Cho, K.H. Positive- and negative-feedback regulations coordinate the dynamic behavior of the Ras-Raf-MEK-ERK signal transduction pathway. J. Cell Sci. 2009, 122 Pt 3, 425-435. [CrossRef]

63. Dong, C.; Waters, S.B.; Holt, K.H.; Pessin, J.E. SOS phosphorylation and disassociation of the Grb2-SOS complex by the ERK and JNK signaling pathways. J. Biol. Chem. 1996, 271, 6328-6332.

64. Xu, B.E.; Wilsbacher, J.L.; Collisson, T.; Cobb, M.H. The N-terminal ERK-binding site of MEK1 is required for efficient feedback phosphorylation by ERK2 in vitro and ERK activation in vivo. J. Biol. Chem. 1999, 274, 34029-34035. [CrossRef] [PubMed] 
65. Ekerot, M.; Stavridis, M.P.; Delavaine, L.; Mitchell, M.P.; Staples, C.; Owens, D.M.; Keenan, I.D.; Dickinson, R.J.; Storey, K.G.; Keyse, S.M. Negative-feedback regulation of FGF signalling by DUSP6/MKP-3 is driven by ERK1/2 and mediated by Ets factor binding to a conserved site within the DUSP6/MKP-3 gene promoter. Biochem. J. 2008, 412, 287-298. [CrossRef] [PubMed]

66. Nolen, B.; Taylor, S.; Ghosh, G. Regulation of protein kinases; controlling activity through activation segment conformation. Mol. Cell 2004, 15, 661-675. [CrossRef] [PubMed]

67. Kornev, A.P.; Haste, N.M.; Taylor, S.S.; Eyck, L.F. Surface comparison of active and inactive protein kinases identifies a conserved activation mechanism. Proc. Natl. Acad. Sci. USA 2006, 103, 17783-17788. [CrossRef]

68. Kornev, A.P.; Taylor, S.S. Dynamics-Driven Allostery in Protein Kinases. Trends Biochem. Sci. 2015, 40, 628-647. [CrossRef]

69. Beenstock, J.; Mooshayef, N.; Engelberg, D. How Do Protein Kinases Take a Selfie (Autophosphorylate)? Trends Biochem. Sci. 2016, 41, 938-953. [CrossRef]

70. Shi, F.; Telesco, S.E.; Liu, Y.; Radhakrishnan, R.; Lemmon, M.A. ErbB3/HER3 intracellular domain is competent to bind ATP and catalyze autophosphorylation. Proc. Natl. Acad. Sci. USA 2010, 107, 7692-7697. [CrossRef]

71. Pike, A.C.; Rellos, P.; Niesen, F.H.; Turnbull, A.; Oliver, A.W.; Parker, S.A.; Turk, B.E.; Pearl, L.H.; Knapp, S. Activation segment dimerization: A mechanism for kinase autophosphorylation of non-consensus sites. EMBO J. 2008, 27, 704-714. [CrossRef]

72. Seger, R.; Ahn, N.G.; Boulton, T.G.; Yancopoulos, G.D.; Panayotatos, N.; Radziejewska, E.; Ericsson, L.; Bratlien, R.L.; Cobb, M.H.; Krebs, E.G. Microtubule-associated protein 2 kinases, ERK1 and ERK2, undergo autophosphorylation on both tyrosine and threonine residues: Implications for their mechanism of activation. Proc. Natl. Acad. Sci. USA 1991, 88, 6142-6146. [CrossRef] [PubMed]

73. Levin-Salomon, V.; Kogan, K.; Ahn, N.G.; Livnah, O.; Engelberg, D. Isolation of intrinsically active (MEK-independent) variants of the ERK family of mitogen-activated protein (MAP) kinases. J. Biol. Chem. 2008, 283, 34500-34510. [CrossRef] [PubMed]

74. Pegram, L.M.; Liddle, J.C.; Xiao, Y.; Hoh, M.; Rudolph, J.; Iverson, D.B.; Vigers, G.P.; Smith, D.; Zhang, H.; Wang, W.; et al. Activation loop dynamics are controlled by conformation-selective inhibitors of ERK2. Proc. Natl. Acad. Sci. USA 2019, 116, 15463-15468. [CrossRef] [PubMed]

75. Sang, D.; Pinglay, S.; Wiewiora, R.P.; Selvan, M.E.; Lou, H.J.; Chodera, J.D.; Turk, B.E.; Gumus, Z.H.; Holt, L.J. Ancestral reconstruction reveals mechanisms of ERK regulatory evolution. eLife 2019, 8, e38805. [CrossRef] [PubMed]

76. Emrick, M.A.; Lee, T.; Starkey, P.J.; Mumby, M.C.; Resing, K.A.; Ahn, N.G. The gatekeeper residue controls autoactivation of ERK2 via a pathway of intramolecular connectivity. Proc. Natl. Acad. Sci. USA 2006, 103, 18101-18106. [CrossRef] [PubMed]

77. Brunner, D.; Oellers, N.; Szabad, J.; Biggs, W.H.; Zipursky, S.L.; Hafen, E. A gain-of-function mutation in Drosophila MAP kinase activates multiple receptor tyrosine kinase signaling pathways. Cell 1994, 76, 875-888. [CrossRef]

78. Goshen-Lago, T.; Goldberg-Carp, A.; Melamed, D.; Darlyuk-Saadon, I.; Bai, C.; Ahn, N.G.; Admon, A.; Engelberg, D. Variants of the yeast MAPK Mpk1 are fully functional independently of activation loop phosphorylation. Mol. Biol. Cell 2016, 27, 2771-2783. [CrossRef]

79. Dhillon, A.S.; Hagan, S.; Rath, O.; Kolch, W. MAP kinase signalling pathways in cancer. Oncogene 2007, 26, 3279-3290. [CrossRef]

80. Hanahan, D.; Weinberg, R.A. Hallmarks of cancer: The next generation. Cell 2011, 144, 646-674. [CrossRef]

81. Samatar, A.A.; Poulikakos, P.I. Targeting RAS-ERK signalling in cancer: Promises and challenges. Nat. Rev. Drug Discov. 2014, 13, 928-942. [CrossRef]

82. Smorodinsky-Atias, K.; Goshen-Lago, T.; Goldberg-Carp, A.; Melamed, D.; Shir, A.; Mooshayef, N.; Beenstock, J.; Karamansha, Y.; DArlyuk-Saadon, I.; Livnah, O.; et al. Intrinsically active variants of Erk oncogenically transform cells and disclose unexpected autophosphorylation capability that is independent of TEY phosphorylation. Mol. Biol. Cell 2016, 27, 1026-1039. [CrossRef] [PubMed]

83. Kushnir, T.; Bar-Cohen, S.; Mooshayef, N.; Lange, R.; Bar-Sinai, A.; Rozen, H.; Salzberg, A.; Engelberg, D.; Paroush, Z. An Activating Mutation in ERK Causes Hyperplastic Tumors in a scribble Mutant Tissue in Drosophila. Genetics 2019. [CrossRef] [PubMed]

84. Groenendijk, F.H.; Bernards, R. Drug resistance to targeted therapies: Deja vu all over again. Mol. Oncol. 2014, 8, 1067-1083. [CrossRef] [PubMed] 
85. Morris, E.J.; Jha, S.; Restaino, C.R.; Dayananth, P.; Zhu, H.; Cooper, A.; Carr, D.; Deng, Y.; Jin, W.; Black, S.; et al. Discovery of a novel ERK inhibitor with activity in models of acquired resistance to BRAF and MEK inhibitors. Cancer Discov. 2013, 3, 742-750. [CrossRef]

86. Moschos, S.J.; Sullivan, R.J.; Hwu, W.J.; Ramanathan, R.K.; Adjei, A.A.; Fong, P.C.; Shapira-Frommer, R.; Tawbi, H.A.; Rubino, J.; Rush, T.S.; et al. Development of MK-8353, an orally administered ERK1/2 inhibitor, in patients with advanced solid tumors. JCI Insight 2018, 3, e92352. [CrossRef]

87. Germann, U.A.; Furey, B.F.; Markland, W.; Hoover, R.R.; Aronov, A.M.; Roix, J.J.; Hale, M.; Boucher, D.M.; Sorrell, D.A.; Martinez-Botella, G.; et al. Targeting the MAPK Signaling Pathway in Cancer: Promising Preclinical Activity with the Novel Selective ERK1/2 Inhibitor BVD-523 (Ulixertinib). Mol. Cancer Ther. 2017, 16, 2351-2363. [CrossRef]

88. Blake, J.F.; Burkard, M.; Chan, J.; Chen, H.; Chou, K.J.; Diaz, D.; Dudley, D.A.; Gaudino, J.J.; Gould, S.E.; Grina, J.; et al. Discovery of (S)-1-(1-(4-Chloro-3-fluorophenyl)-2-hydroxyethyl)-4-(2-((1-methyl-1H-pyrazol-5-y 1)amino)pyrimidin-4-yl)pyridin-2(1H)-one (GDC-0994), an Extracellular Signal-Regulated Kinase 1/2 (ERK1/2) Inhibitor in Early Clinical Development. J. Med. Chem. 2016, 59, 5650-5660. [CrossRef]

89. Aronov, A.M.; Baker, C.; Bemis, G.W.; Cao, J.; Chen, G.; Ford, P.J.; Germann, U.A.; Green, J.; Hale, M.R.; Jacobs, M.; et al. Flipped out: Structure-guided design of selective pyrazolylpyrrole ERK inhibitors. J. Med. Chem. 2007, 50, 1280-1287. [CrossRef]

90. Bhagwat, S.V.; McMillen, W.T.; Cai, S.; Zhao, B.; Whitesell, M.; Kindler, L.; Flack, R.S.; Wu, W.; Huss, K.; Anderson, B.; et al. Discovery of LY3214996, a selective and novel ERK1/2 inhibitor with potent antitumor activities in cancer models with MAPK pathway alterations. Cancer Res. 2017, 77. [CrossRef]

91. Ohori, M.; Kinoshita, T.; Okubo, M.; Sato, K.; Yamazaki, A.; Arakawa, H.; Nishimura, S.; Inamura, N.; Nakajima, H.; Neya, M.; et al. Identification of a selective ERK inhibitor and structural determination of the inhibitor-ERK2 complex. Biochem. Biophys. Res. Commun. 2005, 336, 357-363. [CrossRef]

92. Aronov, A.M.; Tang, Q.; Martinez-Botella, G.; Bemis, G.W.; Cao, J.; Chen, G.; Ewing, N.P.; Ford, P.J.; Germann, U.A.; Green, J.; et al. Structure-guided design of potent and selective pyrimidylpyrrole inhibitors of extracellular signal-regulated kinase (ERK) using conformational control. J. Med. Chem. 2009, 52, 6362-6368. [CrossRef] [PubMed]

93. Herrero, A.; Pinto, A.; Colon-Bolea, P.; Casar, B.; Jones, M.; Agudo-Ibanez, L.; Vidal, R.; Tenbaum, S.P.; Nuciforo, P.; Valdizan, E.M.; et al. Small Molecule Inhibition of ERK Dimerization Prevents Tumorigenesis by RAS-ERK Pathway Oncogenes. Cancer Cell 2015, 28, 170-182. [CrossRef] [PubMed]

94. Ryan, M.B.; Corcoran, R.B. Therapeutic strategies to target RAS-mutant cancers. Nat. Rev. Clin. Oncol. 2018, 15, 709-720. [CrossRef] [PubMed]

95. Brenan, L.; Andreev, A.; Cohen, O.; Pantel, S.; Kamburov, A.; Cacchiarelli, D.; Persky, N.S.; Zhu, C.; Bagul, M.; Goettz, E.M.; et al. Phenotypic Characterization of a Comprehensive Set of MAPK1/ERK2 Missense Mutants. Cell Rep. 2016, 17, 1171-1183. [CrossRef]

96. Goetz, E.M.; Ghandi, M.; Treacy, D.J.; Wagle, N.; Garraway, L.A. ERK mutations confer resistance to mitogen-activated protein kinase pathway inhibitors. Cancer Res. 2014, 74, 7079-7089. [CrossRef]

97. Jha, S.; Morris, E.J.; Hruza, A.; Mansueto, M.S.; Schroeder, G.K.; Arbanas, J.; McMasters, D.; Restaino, C.R.; Dayanath, P.; Black, S.; et al. Dissecting Therapeutic Resistance to ERK Inhibition. Mol. Cancer Ther. 2016, 15, 548-559. [CrossRef]

98. Askari, N.; Diskin, R.; Avitzour, M.; Yaakov, G.; Livnah, O.; Engelberg, D. MAP-quest: Could we produce constitutively active variants of MAP kinases? Mol. Cell. Endocrinol. 2006, 252, 231-240. [CrossRef]

99. Cowley, S.; Paterson, H.; Kemp, P.; Marshall, C.J. Activation of Map Kinase Kinase Is Necessary and Sufficient for Pc12 Differentiation and for Transformation of Nih 3t3 Cells. Cell 1994, 77, 841-852. [CrossRef]

100. Huang, S.; Jiang, Y.; Li, Z.; Nishida, E.; Mathias, P.; Lin, S.; Ulevitch, R.J.; Nemerow, G.R.; Hanj, J. Apoptosis signaling pathway in T cells is composed of ICE/Ced-3 family proteases and MAP kinase kinase 6b. Immunity 1997, 6, 739-749. [CrossRef]

101. Prowse, C.N.; Deal, M.S.; Lew, J. The complete pathway for catalytic activation of the mitogen-activated protein kinase, ERK2. J. Biol. Chem. 2001, 276, 40817-40823. [CrossRef]

102. Robbins, D.J.; Zhen, E.; Owaki, H.; Vanderblit, C.A.; Ebert, D.; Geppert, T.D.; Cobb, M.H. Regulation and properties of extracellular signal-regulated protein kinases 1 and 2 in vitro. J. Biol. Chem. 1993, 268, 5097-5106. [PubMed] 
103. Emrick, M.A.; Hoofnagle, A.N.; Miller, A.S.; Ten Eyck, L.F.; Ahn, N.G. Constitutive activation of extracellular signal-regulated kinase 2 by synergistic point mutations. J. Biol. Chem. 2001, 276, 46469-46479. [CrossRef] [PubMed]

104. Brill, J.A.; Elion, E.A.; Fink, G.R. A role for autophosphorylation revealed by activated alleles of FUS3, the yeast MAP kinase homolog. Mol. Biol. Cell 1994, 5, 297-312. [CrossRef] [PubMed]

105. Madhani, H.D.; Styles, C.A.; Fink, G.R. MAP kinases with distinct inhibitory functions impart signaling specificity during yeast differentiation. Cell 1997, 91, 673-684. [CrossRef]

106. Bott, C.M.; Thorneycroft, S.G.; Marshall, C.J. The sevenmaker gain-of-function mutation in p42 MAP kinase leads to enhanced signalling and reduced sensitivity to dual specificity phosphatase action. FEBS Lett. 1994, 352, 201-205. [CrossRef]

107. Hall, J.P.; Cherkasova, V.; Elion, E.; Gustin, M.C.; Winter, E. The osmoregulatory pathway represses mating pathway activity in Saccharomyces cerevisiae: Isolation of a FUS3 mutant that is insensitive to the repression mechanism. Mol. Cell. Biol. 1996, 16, 6715-6723. [CrossRef]

108. Mutlak, M.; Schlesinger-Laufer, M.; Haas, T.; Shofti, R.; Ballan, N.; Lewis, Y.E.; Zuler, M.; Zohar, Y.; Caspi, L.H.; Kehat, I. Extracellular signal-regulated kinase (ERK) activation preserves cardiac function in pressure overload induced hypertrophy. Int. J. Cardiol. 2018, 270, 204-213. [CrossRef]

109. Canagarajah, B.J.; Khokhlatchev, A.; Cobb, M.H.; Golsdmisth, E.J. Activation mechanism of the MAP kinase ERK2 by dual phosphorylation. Cell 1997, 90, 859-869. [CrossRef]

110. Taylor, C.A.; Cormier, K.W.; Keenan, S.E.; Earnest, S.; Stippec, S.; Wichaidit, C.; Juang, Y.C.; Wang, J.; Shvartsman, S.Y.; Goldsmith, E.J.; et al. Functional divergence caused by mutations in an energetic hotspot in ERK2. Proc. Natl. Acad. Sci. USA 2019, 116, 15514-15523. [CrossRef]

111. Lee, T.; Hoofnagle, A.N.; Kabuyama, Y.; Stoud, J.; Min, X.; Goldsmith, E.J.; Chen, L.; Resing, K.A.; Ahn, N.G. Docking motif interactions in MAP kinases revealed by hydrogen exchange mass spectrometry. Mol. Cell 2004, 14, 43-55. [CrossRef]

112. Dimitri, C.A.; Dowdle, W.; MacKeiga, J.P.; Blenis, J.; Murphy, L.O. Spatially separate docking sites on ERK2 regulate distinct signaling events in vivo. Curr. Biol. 2005, 15, 1319-1324. [CrossRef]

113. Misiura, M.; Kolomeisky, A.B. Kinetic network model to explain gain-of-function mutations in ERK2 enzyme. J. Chem. Phys. 2019, 150, 155101. [CrossRef] [PubMed]

114. Barr, D.; Oashi, T.; Burkhard, K.; Lucius, S.; Samadani, R.; Zhang, J.; Shapiro, P.; MacKerell, A.D.; van der Vaart, A. Importance of domain closure for the autoactivation of ERK2. Biochemistry 2011, 50, 8038-8048. [CrossRef] [PubMed]

115. Mahalingam, M.; Arvind, R.; Ida, H.; Murugan, A.K.; Yamaguchi, M.; Tsuchida, N. ERK2 CD domain mutation from a human cancer cell line enhanced anchorage-independent cell growth and abnormality in Drosophila. Oncol. Rep. 2008, 20, 957-962. [PubMed] 